\title{
An Easy Synthesis of 3,5-Disubstituted 1,2,4-Oxadiazoles from Carboxylic Acids and Arylamidoximes Mediated by Ethyl Chloroformate
}

\author{
Ricardo A. W. Neves Filho, Natércia M. M. Bezerra," José M. Guedes and Rajendra M. Srivastava* \\ Departamento de Química Fundamental, Universidade Federal de Pernambuco, Cidade Universitária, \\ 50740-540 Recife-PE, Brazil
}

\begin{abstract}
Uma síntese limpa, fácil e eficiente de vários 1,2,4-oxadiazóis 3,5-dissubstituídos partindo de um anidrido misto (gerado a partir de um ácido carboxílico e cloroformiato de etila) e amidoxima é descrita.

An efficient, clean and easy high-yielding synthesis of 3,5-disubstituted 1,2,4-oxadiazoles starting from mixed anhydrides (generated from carboxylic acids and ethyl chloroformate) and arylamidoximes is described.
\end{abstract}

Keywords: carboxylic acids, arylamidoximes, 1,2 ,4-oxadiazoles, ${ }^{1} \mathrm{H}$ and ${ }^{13} \mathrm{C}$ NMR spectra

\section{Introduction}

1,2,4-Oxadiazoles are well-known nitrogen compounds and sizeable work has been done in this area since their first preparation in $1884 .{ }^{1}$ A recent review covering the research papers published from 1996 through 2007 describes the interesting synthetic developments of 1,2,4- and 1,3,4-oxadiazoles. ${ }^{2}$ This review also quotes the already established biological attributes to this class of compounds. Although much attention has been given for pharmacological evaluations of 1,2,4-oxadiazoles, recent publications showed also their applicability in the field of luminescent liquid crystals, materials for optical devices, and charge-transporters for organic light-emitting diodes (OLEDs). ${ }^{3}$ Because of the vast importance of this class of compounds, ${ }^{3}$ which is constantly growing, ${ }^{4}$ we decided to develop their simpler and less time-consuming synthesis.

The most prevalent method for synthesizing 1,2,4-oxadiazoles involves $O$-acylation of amidoximes followed by cyclodehydratation. Acyl chlorides, anhydrides, esteres, and trichloroalkanes are commonly used as acylating agents. ${ }^{5}$ Carboxylic acids in the presence of coupling reagents like DCC, DIC or EDC are also employed to achieve the same goal. ${ }^{5,6}$ These procedures need much work to purify the desired 1,2,4-oxadiazoles. Besides

*e-mail: rms_indu@yahoo.com

\#Taken in part from the MSc Dissertation (2007) of Natércia M. M. Bezerra, Universidade Federal de Pernambuco, Recife-PE, Brazil undesired side products are also formed which require, additional time and efforts for their separation.

One-pot methodologies for organic synthesis have attracted chemists' and pharmacists' interest from industries and academia, because these procedures allow reaching the target compounds without isolation of synthetic intermediates. ${ }^{7}$ In addition, one-pot reactions also reduce the use of solvents, reagents and adsorbents commonly employed for purifying the intermediates, being considered green protocols. ${ }^{7}$ Therefore, we have focused our attention in developing a clean one-pot protocol which allows the synthesis of 1,2,4-oxadiazoles in good yields with less work-up and avoids side product formation. Herein, we would like to report, for the first time, the synthesis of some 2,5-disubstituted 1,2,4-oxadiazoles from carboxylic acids and arylamidoximes in the presence of ethyl chloroformate as a coupling agent (Scheme 1 and Table 1).

\section{Results and Discussion}

Although ethyl chloroformate $\mathbf{2}$ has been used in the presence of a base as carbonyl activator reagent for the onepot synthesis of esters and amides, ${ }^{8}$ it has not been utilized in the synthesis of 1,2,4-oxadiazoles. Therefore, first we carried out various reactions involving benzoic acid $\mathbf{1 d}$ and benzamidoxime $\mathbf{4 h}$ by using different organic solvents and bases to standardize the reaction conditions. The best result was found when we used $\mathrm{CH}_{2} \mathrm{Cl}_{2}$ as solvent and $\mathrm{K}_{2} \mathrm{CO}_{3}$ 


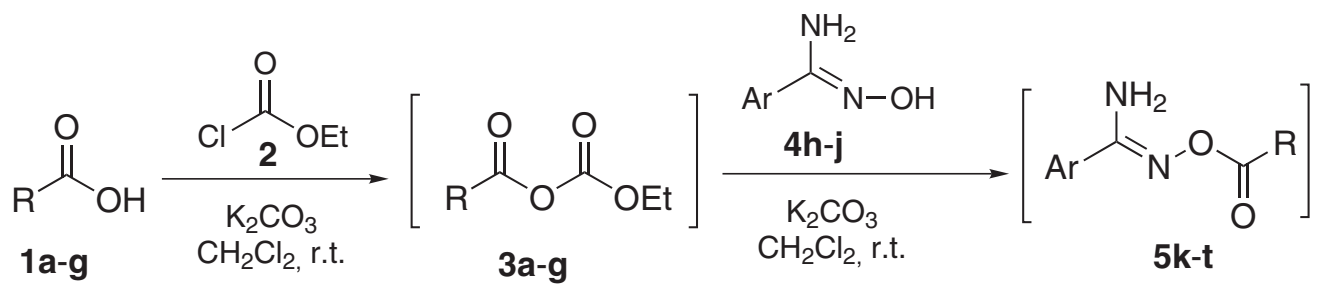

R $\quad \operatorname{Ar}$
a: $\mathrm{C}_{13} \mathrm{H}_{27}$
h: $\mathrm{C}_{6} \mathrm{H}_{5}$
k: $\mathrm{R}=\mathrm{C}_{13} \mathrm{H}_{27}, \mathrm{Ar}=4-\mathrm{CH}_{3} \mathrm{C}_{6} \mathrm{H}_{4}$
b: $\mathrm{C}_{17} \mathrm{H}_{35}$
i: $4-\mathrm{CH}_{3} \mathrm{C}_{6} \mathrm{H}_{4}$
I: $\quad \mathrm{R}=\mathrm{C}_{13} \mathrm{H}_{27}, \mathrm{Ar}=4-\mathrm{ClC}_{6} \mathrm{H}_{4}$
c: $\mathrm{C}_{15} \mathrm{H}_{31}$
j: $4-\mathrm{ClC}_{6} \mathrm{H}_{4}$
m: $\mathrm{R}=\mathrm{C}_{17} \mathrm{H}_{35}, \mathrm{Ar}=4-\mathrm{CH}_{3} \mathrm{C}_{6} \mathrm{H}_{4}$
d: $\mathrm{C}_{6} \mathrm{H}_{5}$
n: $\mathrm{R}=\mathrm{C}_{17} \mathrm{H}_{35}, \mathrm{Ar}=4-\mathrm{ClC}_{6} \mathrm{H}_{4}$
e: $4-\mathrm{CH}_{3} \mathrm{C}_{6} \mathrm{H}_{4}$
o: $\mathrm{R}=\mathrm{C}_{15} \mathrm{H}_{31}, \quad \mathrm{Ar}=4-\mathrm{CH}_{3} \mathrm{C}_{6} \mathrm{H}_{4}$
f: $\mathrm{C}_{6} \mathrm{H}_{11}$
p: $\mathrm{R}=4-\mathrm{CH}_{3} \mathrm{C}_{6} \mathrm{H}_{4}, \mathrm{Ar}=4-\mathrm{CH}_{3} \mathrm{C}_{6} \mathrm{H}_{4}$

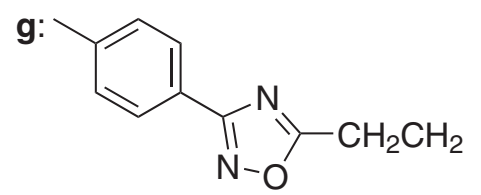
q: $\mathrm{R}=\mathrm{C}_{6} \mathrm{H}_{5}, \mathrm{Ar}=\mathrm{C}_{6} \mathrm{H}_{5}$
r: $\mathrm{R}=\mathrm{C}_{6} \mathrm{H}_{5}, \mathrm{Ar}=4-\mathrm{CH}_{3} \mathrm{C}_{6} \mathrm{H}_{4}$

Dry heating

$120^{\circ} \mathrm{C}$

s: $\mathrm{R}=\mathrm{C}_{6} \mathrm{H}_{11}, \mathrm{Ar}=4-\mathrm{CH}_{3} \mathrm{C}_{6} \mathrm{H}_{4}$<smiles>CCc1nc(-c2ccc(C)cc2)no1</smiles>

$\mathrm{Ar}=4-\mathrm{CH}_{3} \mathrm{C}_{6} \mathrm{H}_{4}$

Scheme 1.

or $\mathrm{Et}_{3} \mathrm{~N}$ as bases. We chose to use potassium carbonate because it is cheap as well as easy to remove after the reaction. Thus, a suitable carboxylic acid 1a-g was stirred in the presence of $\mathrm{K}_{2} \mathrm{CO}_{3}$ in $\mathrm{CH}_{2} \mathrm{Cl}_{2}$ for 30 min to achieve the formation of carboxylic acid potassium salt which reacts with ethyl chloroformate $\mathbf{2}$ to generate in situ the mixed anhydrides 3a-g. Then, an appropriate amidoxime 4h-j was added to the same solution followed by stirring for an additional $2 \mathrm{~h}$. The reaction between $\mathbf{3 a - g}$ and $\mathbf{4 h}-\mathbf{j}$ forms $O$-acylamidoximes $\mathbf{5 k - \mathbf { t }}$ with the liberation of $\mathrm{CO}_{2}$ and $\mathrm{EtOH}$. Although the intermediates $\mathbf{5 k}$ - $\mathbf{t}$ can be isolated and characterized, we avoided their isolation in many cases. In fact, these were cyclodehydrated individually to afford 1,2,4-oxadiazoles (Table 1).

In order to verify the structure of the intermediates $\mathbf{5 k - t}$, we have isolated two known products $\mathbf{5 p}$ and $\mathbf{5 q}$ whose physical and chemical properties agreed with the literature. ${ }^{10}$ Once the structures of the above-cited intermediates have been established, we proceeded to obtain the final products in excellent yields (75-93\%). This general protocol has worked well with aromatic, aliphatic and carbocyclic carboxylic acids (entries 1-10, Table 1). Compounds 5k-o,t are new ones and their structures have been confirmed by, IR, ${ }^{1} \mathrm{H}$ and ${ }^{13} \mathrm{C}$ NMR spectra and elemental analyses. The known compounds 5p-r,s were characterized by comparing their reported melting points and spectral data. ${ }^{9}$

\section{Conclusions}

In summary, we have developed an alternate new method to synthesize 1,2,4-oxadiazoles from carboxylic acids and arylamidoximes using ethyl chloroformate as a coupling agent. The desired 3,5-disubstituted oxadiazoles 6k-t have been obtained in excellent yields after simple work-up. This protocol is applicable for synthesizing 1,2,4-oxadiazoles containing aryl or alkyl groups attached at their C-5 side-chain. Further, this procedure is also suitable for the obtaining bis-1,2,4-oxadiazoles.

\section{Experimental}

\section{General experimental procedures}

All reagents were obtained from commercial sources and used without further purification. Infrared spectra were recorded on a Perkin-Elmer model 283 spectrometer in $\mathrm{KBr}$ discs. ${ }^{1} \mathrm{H}$ and ${ }^{13} \mathrm{C}$ NMR spectra of $\mathrm{CDCl}_{3}$ solutions were obtained in a Varian 300-MHz instrument using 
Table 1. Synthesis of 3,5-disubstituted 1,2,4-oxadiazoles 6k-t

\begin{tabular}{|c|c|c|c|c|}
\hline Entry & Compound & Yield / (\%) & $\mathrm{mp} /\left({ }^{\circ} \mathrm{C}\right)$ & mp $[\text { Lit. }]^{9} /\left({ }^{\circ} \mathrm{C}\right)$ \\
\hline 1 & & 86 & 45 & - \\
\hline 2 & & 80 & 51 & - \\
\hline 3 & & 83 & 56 & - \\
\hline 4 & & 79 & 53 & - \\
\hline 5 & & 90 & 57 & 57.1 \\
\hline 6 & & 75 & $133-134$ & 134 \\
\hline 7 & & 86 & 108-109 & 110 \\
\hline 8 & & 93 & $105-106$ & $105-106$ \\
\hline 9 & & 91 & 55 & - \\
\hline 10 & & 84 & $169-170$ & $171-172$ \\
\hline
\end{tabular}


tetramethylsilane (TMS) as the internal standard. Elemental analysis was performed with a Carlo Erba instrument model E-1110. Carboxylic acids 1a-f were obtained from commercial sources while $\mathbf{1 g}$ and arylamidoximes 4h-j were prepared following the procedures reported earlier. ${ }^{9,11}$

Typical experimental procedure

A suitable carboxylic acid $\mathbf{1 a - f}(1.6 \mathrm{mmol})$ was dissolved in dry $\mathrm{CH}_{2} \mathrm{Cl}_{2}(8.0 \mathrm{~mL})$ and placed in a round bottom flask followed by the addition of $\mathrm{K}_{2} \mathrm{CO}_{3}(0.33 \mathrm{~g}$, $2.4 \mathrm{mmol}$ ) under stirring and kept as such for $30 \mathrm{~min}$ at room temperature. Later, ethyl chloroformate $2(0.2 \mathrm{~mL}$, $2.4 \mathrm{mmol}$ ) was added to the same flask and stirred for an additional $30 \mathrm{~min}$. Finally, the addition of an appropriate amidoxime $4 \mathbf{h}-\mathbf{j}$ ( $1.6 \mathrm{mmol}$ ) with continuous agitation for $2 \mathrm{~h}$ completed the reaction. Filtration and solvent evaporation under reduced pressure furnished the crude product which upon heating in an oil bath at $120^{\circ} \mathrm{C}$ for $4 \mathrm{~h}$ gave the desired compounds which were crystallized from $\mathrm{EtOH}$.

\section{3-(4-Tolyl)-5-tridecanyl-1,2,4-oxadiazole (6k)}

Yield: 86\%; colorless crystals; mp $45{ }^{\circ} \mathrm{C}$; $\mathrm{R}_{\mathrm{f}} 0.61$ $\left(\mathrm{CHCl}_{3}: \mathrm{C}_{6} \mathrm{H}_{12}, 1: 1\right)$. IR (KBr) $v_{\max } / \mathrm{cm}^{-1}: 3049,2922,2926$, 2846, 1576. ${ }^{1} \mathrm{H}$ NMR (300 MHz, $\left.\mathrm{CDCl}_{3}\right) ; \delta 0.87$ (t, J 7.5 $\mathrm{Hz}, 3 \mathrm{H}$ ), 1.25-1.48 (bs, 22H ), 1.86 (quintet, $J 7.5 \mathrm{~Hz}, 2 \mathrm{H}$ ), 2.93 (t, J 7.5 Hz, 2H), 2.41 (s, 3H), 7.23 (d, J $8.4 \mathrm{~Hz}, 2 \mathrm{H})$, $7.96(\mathrm{~d}, J 8.4 \mathrm{~Hz}, 2 \mathrm{H}) .{ }^{13} \mathrm{C}$ NMR $\left(75 \mathrm{MHz}, \mathrm{CDCl}_{3}\right) ; \delta 14.13$, 21.56, 22.68, 26.67, 29.03, 29.34, 29.54, 29.62, 31.90, 124.07, 127.27, 129.51, 141.34, 168.51, 179.91. Anal. Calc. for $\mathrm{C}_{22} \mathrm{H}_{36} \mathrm{~N}_{2} \mathrm{O} \mathrm{C}, 76.69 ; \mathrm{H}, 10.53 ; \mathrm{N}, 8.13$. Found: C, 76.43; H, 10.98; N, 8.21.

\section{3-(4-Chlorophenyl)-5-tridecanyl-1,2,4-oxadiazole (6l)}

Yield: 80\%; colorlerss crystals; mp $51{ }^{\circ} \mathrm{C}$; $\mathrm{R}_{\mathrm{f}} 0.62$ $\left(\mathrm{CHCl}_{3}: \mathrm{C}_{6} \mathrm{H}_{12}, 1: 1\right)$. IR (KBr) $v_{\max } / \mathrm{cm}^{-1}: 3049 ; 2920$; $2925 ; 2843 ; 1572 ;{ }^{1} \mathrm{H}$ NMR $\left(300 \mathrm{MHz}, \mathrm{CDCl}_{3}\right) ; \delta 0.88(\mathrm{t}$, $J 7.2 \mathrm{~Hz}, 3 \mathrm{H}$ ), 1.25-1.49 (bs, 22H ), 1.86 (quintet, $J 7.8$ $\mathrm{Hz}, 2 \mathrm{H}), 2.93$ (t, J 7.5 Hz, 2H), 7.45 (d, J $8.4 \mathrm{~Hz}, 2 \mathrm{H})$, $8.01(\mathrm{~d}, J 8.7 \mathrm{~Hz}, 2 \mathrm{H}) .{ }^{13} \mathrm{C} \mathrm{NMR}\left(75 \mathrm{MHz}, \mathrm{CDCl}_{3}\right) ; \delta 14.1$, 22.7, 26.6, 29.0, 29.1, 29.3, 29.5, 29.6, 29.65, 29.68, 31.9, 125.4, 128.7, 129.1, 137.2, 167.4, 180.3. Anal. Calc. for $\mathrm{C}_{21} \mathrm{H}_{33} \mathrm{ClN}_{2} \mathrm{O} \mathrm{C}, 69.11 ; \mathrm{H}, 9.11 ; \mathrm{N}, 7.68$. Found: C, 69.21; H, 9.54; N, 7.58.

\section{5-Heptadecanyl-3-p-tolyl-1,2,4-oxadiazole (6m)}

Yield: 83\%; colorless crystals; mp $56{ }^{\circ} \mathrm{C} ; \mathrm{R}_{\mathrm{f}} 0.60$ $\left(\mathrm{CHCl}_{3}: \mathrm{C}_{6} \mathrm{H}_{12}, 1: 1\right)$. IR (KBr) $v_{\max } / \mathrm{cm}^{-1}: 3049,2921,2846$, 1576. ${ }^{1} \mathrm{H}$ NMR $\left(300 \mathrm{MHz} \mathrm{CDCl}_{3}\right) \delta 0.87(\mathrm{t}, J 5.4 \mathrm{~Hz}, 3 \mathrm{H})$, 1.25-1.50 (bs, $28 \mathrm{H}$ ), 1.86 (quintet, $J 6.9 \mathrm{~Hz}, 2 \mathrm{H}$ ), 2.41 (s,
$3 \mathrm{H}), 2.93(\mathrm{t}, J 6.9 \mathrm{~Hz}, 2 \mathrm{H}), 7.23(\mathrm{~d}, J 8.1 \mathrm{~Hz}, 2 \mathrm{H}), 7.96(\mathrm{~d}$, $J 8.1 \mathrm{~Hz}, 2 \mathrm{H}) .{ }^{13} \mathrm{C} \mathrm{NMR}\left(75 \mathrm{MHz}, \mathrm{CDCl}_{3}\right) ; \delta 14.1,22.7$, 26.7, 29.0, 29.3, 29.5, 29.6, 31.9, 125.4, 128.7, 129.1, 137.2, 167.4, 180.3. Anal. Calc. for $\mathrm{C}_{26} \mathrm{H}_{42} \mathrm{~N}_{2} \mathrm{O}$ C, 78.34; H, 10.62; N, 7.03. Found: C, 78.68; H, 10.58, N, 7.21\%.

3-(4-Chlorophenyl)-5-heptadecanyl-1,2,4-oxadiazole (6n)

Yield: 79\%; colorless crystals; $\mathrm{mp} 53{ }^{\circ} \mathrm{C} ; \mathrm{R}_{\mathrm{f}} 0.53$. $\left(\mathrm{CHCl}_{3}: \mathrm{C}_{6} \mathrm{H}_{12}, 1: 1\right)$. IR (KBr) $v_{\text {max }} / \mathrm{cm}^{-1}: 3048,2925$, 2925, 2847, 1574. ${ }^{1} \mathrm{H}$ NMR (300 $\left.\mathrm{MHz}, \mathrm{CDCl}_{3}\right) \delta 0.88$ (t, $J 6.6 \mathrm{~Hz}, 3 \mathrm{H}$ ), 1.25-1.50 (bs, $28 \mathrm{H}$ ), 1.86 (quintet, $J 8.1$ $\mathrm{Hz}, 2 \mathrm{H}), 2.94$ (t, J $8.1 \mathrm{~Hz}, 2 \mathrm{H}), 7.45$ (d, J $8.7 \mathrm{~Hz}, 2 \mathrm{H})$, $8.01(\mathrm{~d}, J 8.7 \mathrm{~Hz}, 2 \mathrm{H}) .{ }^{13} \mathrm{C} \mathrm{NMR}\left(75 \mathrm{MHz}, \mathrm{CDCl}_{3}\right) ; \delta 14.1$, 22.7, 26.6, 29.0, 29.1, 29.3, 29.5, 29.6, 29.64, 29.67, 31.9, 125.4, 128.7, 129.1, 137.2, 167.4, 180.3. Anal. Calc. for $\mathrm{C}_{25} \mathrm{H}_{39} \mathrm{ClN}_{2} \mathrm{O}$ C, 71.66; H, 9.38; N, 6.69. Found: C, 72.01; $\mathrm{H}, 9.47$;, $6.98 \%$.

\section{5-Pentadecanyl-3-p-tolyl-1,2,4-oxadiazole (6o)}

Yield: 90\%; colorless crystals; mp $57{ }^{\circ} \mathrm{C}$ (lit, ${ }^{9} \mathrm{mp}$ $\left.57.1^{\circ} \mathrm{C}\right) ; \mathrm{R}_{\mathrm{f}} 0.51\left(\mathrm{CHCl}_{3}: \mathrm{C}_{6} \mathrm{H}_{12}, 1: 1\right)$. IR $(\mathrm{KBr}) \mathrm{v}_{\max } / \mathrm{cm}^{-1}$ : 3063, 2954, 2917, 2848, 1588. ${ }^{1} \mathrm{H}$ NMR (300 MHz, $\mathrm{CDCl}_{3}$ ); $\delta 0.88(\mathrm{t}, J 6.9 \mathrm{~Hz}, 3 \mathrm{H}), 1.25-1.44$ (bs, $24 \mathrm{H}$ ), 1.86 (quintet, $J 7.8 \mathrm{~Hz}, 2 \mathrm{H}), 2.41$ (s, 3H), 2.93 (t, J 7.8 Hz, 2H), 7.23 (d, $J 8.1 \mathrm{~Hz}, 2 \mathrm{H}), 7.95(\mathrm{~d}, J 8.1 \mathrm{~Hz}, 2 \mathrm{H})$.

\section{3,5-Di-p-tolyl-1,2,4-oxadiazole (6p)}

Yield: 75\%; colorless crystals; mp $133{ }^{\circ} \mathrm{C}$ (lit, ${ }^{9} \mathrm{mp} 134$ $\left.{ }^{\circ} \mathrm{C}\right) ; \mathrm{R}_{\mathrm{f}} 0.72\left(\mathrm{CHCl}_{3}\right)$. IR (KBr) $v_{\max } / \mathrm{cm}^{-1}: 3023,2920,2850$, 1594. ${ }^{1} \mathrm{H}$ NMR (300 MHz, $\mathrm{CDCl}_{3}$ ); $\delta 2.39$ and 2.40 (2s, $6 \mathrm{H}), 7.27$ (d, J 7.6 Hz, 2H, ), 7.28 (d, J 7.8 Hz, 2H), 8.05 (d, J 7.8 Hz, 2H, ), 8.06 (d, J 7.6 Hz, 2H).

\section{3,5-Diphenyl-1,2,4-oxadiazole (6q)}

Yield: 86\%; colorless crystals; mp 108-109 ${ }^{\circ} \mathrm{C}$ (lit. ${ }^{9} \mathrm{mp}$ $\left.110{ }^{\circ} \mathrm{C}\right) ; \mathrm{R}_{\mathrm{f}} 0.70\left(\mathrm{CHCl}_{3}\right)$. IR ( $\left.\mathrm{KBr}\right) v_{\max } / \mathrm{cm}^{-1}: 3022,2920$, 2839, 1594. ${ }^{1} \mathrm{H} \mathrm{NMR}\left(300 \mathrm{MHz}, \mathrm{CDCl}_{3}\right) ; \delta$ 7.51-7.62 (m, $6 \mathrm{H}), 8.14-8.25(\mathrm{~m}, 4 \mathrm{H})$.

\section{3-Phenyl-3-p-tolyl-1,2,4-oxadiazole (6r)}

Yield: 93\%; colorless crystals; mp 105-106 ${ }^{\circ} \mathrm{C}$ (lit, ${ }^{9} \mathrm{mp}$ $\left.105-106^{\circ} \mathrm{C}\right) ; \mathrm{R}_{\mathrm{f}} 0.52\left(\mathrm{CHCl}_{3} / \mathrm{C}_{6} \mathrm{H}_{12}, 1: 1\right)$. IR $(\mathrm{KBr}) v_{\max } / \mathrm{cm}^{-1}$ : 3049, 2955, 2915, 1560. ${ }^{1} \mathrm{H}$ NMR (300 MHz, $\left.\mathrm{CDCl}_{3}\right) ; \delta$ $2.44(\mathrm{~s}, 3 \mathrm{H}), 7.32(\mathrm{~d}, J 9.6 \mathrm{~Hz}, 2 \mathrm{H}), 7.55-7.60(\mathrm{~m}, 3 \mathrm{H})$, 8.07 (d, J 8.7 Hz, 2H), 8.22 (d, J 9.6 Hz, 2H).

\section{5-Cyclohexyl-4-p-tolyl-1,2,4-oxadiazole (6s)}

Yield: $91 \%$; colorless crystals; $\mathrm{mp} 55^{\circ} \mathrm{C}$; $\mathrm{R}_{\mathrm{f}} 0.80$ $\left(\mathrm{CHCl}_{3}\right)$. IR $(\mathrm{KBr}) \mathrm{v}_{\max } / \mathrm{cm}^{-1}: 3035,2918,2852,1589 .{ }^{1} \mathrm{H}$ NMR (300 MHz, $\left.\mathrm{CDCl}_{3}\right) ; \delta 1.25-2.15(\mathrm{~m}, 10 \mathrm{H}), 2.41(\mathrm{~s}$, 
$3 \mathrm{H}), 3.00\left(\mathrm{tt}, J_{a x-a x} 11.1 \mathrm{~Hz}, J_{a x-e q} 3.6 \mathrm{~Hz}, 1 \mathrm{H}\right), 7.27(\mathrm{~d}, J 8.4$ $\mathrm{Hz}, 2 \mathrm{H}), 7.96(\mathrm{~d}, J 8.4 \mathrm{~Hz}, 2 \mathrm{H}) .{ }^{13} \mathrm{C} \mathrm{NMR}\left(75 \mathrm{MHz}, \mathrm{CDCl}_{3}\right)$; $\delta$ 21.5, 25.3, 25.5, 30.2, 36.3, 124.2, 127.3, 129.4, 141.2, 168.0, 182.7. Anal. Calc. for $\mathrm{C}_{15} \mathrm{H}_{18} \mathrm{~N}_{2} \mathrm{O}$ C, 74.35; H, 7.49; N, 11.56. Found: C, 74.48; H, 7.33; N, 11.62.

\section{1,2-Bis-(3-p-tolyl-1,2,4-oxadiazol-5-yl)ethane (6t)}

Yield: $84 \%$; colorless crystals; mp $169-170{ }^{\circ} \mathrm{C}$ (lit. ${ }^{9}$ mp 171-172 $\left.{ }^{\circ} \mathrm{C}\right) ; \mathrm{R}_{\mathrm{f}} 0.73\left(\mathrm{CHCl}_{3}\right)$. IR $(\mathrm{KBr}) \mathrm{v}_{\max } / \mathrm{cm}^{-1}$ : 3032, 2926, 2854, 1590. ${ }^{1} \mathrm{H}$ NMR (300 MHz, $\mathrm{CDCl}_{3}$ ); $\delta$ $2.41(\mathrm{~s}, 6 \mathrm{H}), 3.57(\mathrm{~s}, 4 \mathrm{H}), 7.28(\mathrm{~d}, J 8.4 \mathrm{~Hz}, 4 \mathrm{H}), 7.95$ (d, $J 8.4 \mathrm{~Hz}, 4 \mathrm{H})$.

\section{Acknowledgments}

The authors are grateful to the Brazilian National Research Council (CNPq) for financial support. One of us N.M.M.B. is thankful to Coordination for the Improvement of Higher Education Staff (CAPES) for a MSc fellowship.

\section{Supplementary Information}

Detailed experimental procedures and full set of ${ }^{1} \mathrm{H}$ and ${ }^{13} \mathrm{C}$ NMR spectra are available free of charge at http://jbcs.sbq.org.br, as a PDF file.

\section{References}

1. Tiemann, F.; Krüger, P.; Tiemann, F.; Krüger, P.; Ber. Dtsch. Chem. Ges. 1884, 17, 1685.

2. Jakopin, Z.; Dolenc, M. S.; Curr. Org. Chem. 2008, 12, 850.

3. Torgova, S. I.; Karamysheva, L. A.; Strigazzi, A.; Braz. J. Phys. 2002, 32, 593; Gallardo, H.; Cristiano, R.; Vieira, A. A.; Neves Filho, R. A. W.; Srivastava, R. M.; Synthesis 2008, 605; Gallardo, H.; Cristiano, R.; Vieira, A. A.; Neves Filho, R. A. W.; Srivastava, R. M.; Bechtold, I. H.; Liq. Cryst. 2008, 35, 857; Kawamonzen, Y.; Nakayama, T.; Jpn. Kokai Tokkyo Koho 2004, 51; Taguchi, T.; Jpn. Kokai Tokkyo Koho 2000, 11.

4. Rovner, S.; $C \& E N$ 2007, 85, 17; Jarvis, L. M.; $C \& E N$ 2008, $86,15$.

5. Kaboudin, B.; Saadati, F.; J. Heterocycl. Chem. 2005, 42, 699; Srivastava, R. M.; Seabra, G. M.; J. Braz. Chem. Soc. 1997, 8, 397; de Freitas, J. J. R.; de Freitas, J. C. R.; da Silva, L. P.; de Freitas Filho, J. R.; Kimura, G. Y. V.; Srivastava,
R. M.; Tetrahedron Lett. 2007, 48, 6195; Belen'kii, L. I.; Brokhovetskii, D. B.; Krayushkin, M. M.; Tetrahedron 1990, 46, 1659; Denney, D. B.; Feig, G.; J. Am. Chem. Soc. 1959, 81, 225.

6. Katritzky, A. R.; Shestopalov, A. A.; Suzuki, K.; ARKIVOK 2005, 7, 36 and references cited therein; Braga, V. M. L.; Melo, S. J.; Srivastava, R. M.; Falcão, E. P. S.; J. Braz.Chem. Soc. 2004, 15, 603; Melo, S. J.; Sobral, A. D.; Lopes, H. L.; Srivastava, R. M.; J. Braz.Chem. Soc. 1998, 9, 465.

7. Pervin, G.; Jacob, R. G.; Bottselle, G. V.; Kublik, E. L.; Lenardão, E. J.; Cella, R.; Santos, P. C. S.; J. Braz. Chem. Soc. 2005, 16, 857; Bonacorso, A. G.; Oliveira, M. R.; Costa, M. B.; Silva, L. B.; Zanatta, N.; Martins, M. A. P.; Flores, A. F. C.; J. Braz.Chem. Soc. 2005, 16, 868; Lenardão, E. J.; Freitag, R. A.; Dabdoub, J. M.; Batista, A. C. F.; Silveira, C. C.; Quim. Nova 2003, 26, 123.

8. Nakamura, T.; Noguchi, T.; Miyachi, H.; Hashimoto, Y.; Chem. Pharm. Bull. 2007, 55, 651; Jarho, E. M.; Venaelaeinen, J. I.; Poutiainen, S.; Leskinen, H.; Vepsaelaeinen, J.; Christiaans, J. A. M.; Forsberg, M. M.; Maennistoe, P. T.; Wallen, E. A. A.; Bioorg. Med. Chem. 2007, 15, 2024; Hargaden, G. C.; Muller-Bunz, H.; Guiry, P. J.; Eur. J. Org. Chem. 2007, 25, 4235; Kikuchi, M.; Inagaki, T.; Nishiyama, H.; Synlett 2007, 7, 1075; Almasi, D.; Alonso, D. A.; Gomez-Bengoa, E.; Nagel, Y.; Najera, C.; Eur. J. Org. Chem. 2007, 14, 2328.

9. For compounds 6o-r see: Bezerra, N. M. M.; Oliveira, S. P.; Srivastava, R. M.; da Silva, J. R.; Il Farmaco 2005, 60, 955; Leite, L. F. C.; Srivastava, R. M.; Cavalcanti, A. P.; Bull. Soc. Chim. Belges 1989, 98, 203; Goetz, N.; Synthesis 1976, 268; Chiou, S.; J. Heterocycl. Chem. 1989, 26, 125; For compound 6t see: Srivastava, R. M.; da Silva, A. J. C. N.; de Oliveira, M. L.; J. Braz. Chem. Soc. 1993, 4, 84.

10. For compound 5p see Foti, F.; Grassi, G.; Resitiano, F.; Caruso, F.; J. Chem. Res. 1982, 11, 302; For compound 5s see Kawashima, E.; Ando, Y.; Tubei, K.; Miyamae, H.; Heterocycles 1987, 26, 1015.

11. Srivastava, R. M.; Brinn, I. M.; Machuca-Herrera, J. O.; Faria, H. B.; Carpenter, G. B.; Venkatesh, C. G.; de Morais, L. P. F.; J. Mol. Struct. 1997, 406, 159.

Received: October 20, 2008 Web Release Date: July 23, 2009 


\title{
An Easy Synthesis of 3,5-Disubstituted 1,2,4-oxadiazoles from Carboxylic Acids and Arylamidoximes Mediated by Ethyl Chloroformate
}

\author{
Ricardo A. W. Neves Filho, Natércia M. M. Bezerra, ${ }^{\#}$ José M. Guedes and Rajendra M. Srivastava* \\ Departamento de Química Fundamental, Universidade Federal de Pernambuco, Cidade Universitária, \\ 50740-540 Recife-PE, Brazil
}

\section{Experimental}

\section{General experimental procedures}

All reagents were obtained from commercial sources and used without further purification. Infrared spectra were recorded on a Perkin-Elmer model 283 spectrometer in $\mathrm{KBr}$ discs. ${ }^{1} \mathrm{H}$ and ${ }^{13} \mathrm{C}$ NMR spectra of $\mathrm{CDCl}_{3}$ solutions were obtained in a Varian 300-MHz instrument using tetramethylsilane (TMS) as the internal standard. Elemental analysis was performed with a Carlo Erba instrument model E-1110. Carboxylic acids 1a-f were obtained from commercial sources while $\mathbf{1 g}$ and arylamidoximes $\mathbf{4 h}-\mathbf{j}$ were prepared following the procedure reported earlier. ${ }^{1,2}$

\section{Typical experimental procedure}

A suitable carboxylic acid 1a-f $(1.6 \mathrm{mmol})$ was dissolved in dry $\mathrm{CH}_{2} \mathrm{Cl}_{2}(8.0 \mathrm{~mL})$ and placed in a round bottom flask followed by the addition of $\mathrm{K}_{2} \mathrm{CO}_{3}(0.332 \mathrm{~g}, 2.4 \mathrm{mmol})$ under stirring and kept as such for $30 \mathrm{~min}$ at room temperature. Later ethyl chloroformate $2(0.2 \mathrm{~mL}, 2.4 \mathrm{mmol})$ was added to the same flask and stirred for an additional $30 \mathrm{~min}$. Finally, the addition of an appropriate amidoxime $\mathbf{4 h}-\mathbf{j}$ (1.6 mmol) with continuous agitation for $2 \mathrm{~h}$ completed the reaction. Filtration and solvent evaporation under reduced pressure furnished the crude product which upon heating in an oil bath at $120{ }^{\circ} \mathrm{C}$ for $4 \mathrm{~h}$ gave the desired compounds which were crystallized from EtOH.

\section{3-(4-Tolyl)-5-tridecanyl-1,2,4-oxadiazole (6k)}

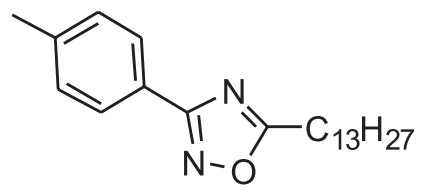

*e-mail: rms_indu@yahoo.com

"Taken in part from the M. Sc. Dissertation (2007) of Natércia M. M Bezerra, Universidade Federal de Pernambuco, Recife, PE, Brazil
Yield: $86 \%$; colorless crystals; $\mathrm{mp} 45{ }^{\circ} \mathrm{C}$; $\mathrm{R}_{\mathrm{f}} 0.61$ $\left(\mathrm{CHCl}_{3}: \mathrm{C}_{6} \mathrm{H}_{12}, 1: 1\right)$. IR (KBr) $v_{\max } / \mathrm{cm}^{-1}: 3049,2922,2926$, 2846, 1576. ${ }^{1} \mathrm{H}$ NMR (300 MHz, $\left.\mathrm{CDCl}_{3}\right) ; \delta 0.87$ (t, J 7.5 $\mathrm{Hz}, 3 \mathrm{H}$ ), 1.25-1.48 (bs, 22H ), 1.86 (quintet, $J 7.5 \mathrm{~Hz}$, $2 \mathrm{H}), 2.93(\mathrm{t}, J 7.5 \mathrm{~Hz}, 2 \mathrm{H}), 2.41(\mathrm{~s}, 3 \mathrm{H}), 7.23(\mathrm{~d}, J 8.4 \mathrm{~Hz}$, 2H), 7.96 (d, $J 8.4 \mathrm{~Hz}, 2 \mathrm{H}) .{ }^{13} \mathrm{C} \mathrm{NMR}\left(75 \mathrm{MHz}, \mathrm{CDCl}_{3}\right)$; $\delta 14.13,21.56,22.68,26.67,29.03,29.34,29.54,29.62$, 31.90, 124.07, 127.27, 129.51, 141.34, 168.51, 179.91. Anal. Calc. for $\mathrm{C}_{22} \mathrm{H}_{36} \mathrm{~N}_{2} \mathrm{O}$ C, 76.69; H, 10.53; N, 8.13. Found: C, 76.43; H, 10.98; N, 8.21.

3-(4-Chlorophenyl)-5-tridecanyl-1,2,4-oxadiazole (6l)<smiles>[3H]Cc1nc(-c2ccc(Cl)cc2)no1</smiles>

Yield: 80\%; colorless crystals; mp $51{ }^{\circ} \mathrm{C} ; \mathrm{R}_{\mathrm{f}} 0.62$ $\left(\mathrm{CHCl}_{3}: \mathrm{C}_{6} \mathrm{H}_{12}, 1: 1\right)$. IR (KBr) $v_{\max } / \mathrm{cm}^{-1}: 3049 ; 2920 ; 2925$; 2843; 1572; ${ }^{1} \mathrm{H}$ NMR (300 MHz, $\left.\mathrm{CDCl}_{3}\right) ; \delta 0.88$ (t, $J 7.2$ $\mathrm{Hz}, 3 \mathrm{H}$ ), 1.25-1.49 (bs, 22H ), 1.86 (quintet, $J 7.8 \mathrm{~Hz}$, 2H), 2.93 (t, J 7.5 Hz, 2H), 7.45 (d, J $8.4 \mathrm{~Hz}, 2 \mathrm{H}), 8.01$ $(\mathrm{d}, J 8.7 \mathrm{~Hz}, 2 \mathrm{H}) .{ }^{13} \mathrm{C} \mathrm{NMR}\left(75 \mathrm{MHz}, \mathrm{CDCl}_{3}\right) ; \delta$ 14.1, 22.7, 26.6, 29.0, 29.1, 29.3, 29.5, 29.6, 29.65, 29.68, 31.9, 125.4, 128.7, 129.1, 137.2, 167.4, 180.3. Anal. Calc. for $\mathrm{C}_{21} \mathrm{H}_{33} \mathrm{ClN}_{2} \mathrm{O}$ C, 69.11; H, 9.11; N, 7.68. Found: C, 69.21; $\mathrm{H}, 9.54 ; \mathrm{N}, 7.58$.

5-Heptadecanyl-3-p-tolyl-1,2,4-oxadiazole (6m)<smiles>[3H]C(c1nc(-c2ccc(C)cc2)no1)C([3H])([3H])[3H]</smiles>

Yield: 83\%; colorless crystals; mp $56{ }^{\circ} \mathrm{C}$; $\mathrm{R}_{\mathrm{f}} 0.60$ $\left(\mathrm{CHCl}_{3}: \mathrm{C}_{6} \mathrm{H}_{12}, 1: 1\right)$. IR (KBr) $v_{\max } / \mathrm{cm}^{-1}: 3049,2921,2846$, 1576. ${ }^{1} \mathrm{H}$ NMR $\left(300 \mathrm{MHz}, \mathrm{CDCl}_{3}\right) \delta 0.87$ (t, J $\left.5.4 \mathrm{~Hz}, 3 \mathrm{H}\right)$, 1.25-1.50 (bs, 28H ), 1.86 (quintet, $J 6.9 \mathrm{~Hz}, 2 \mathrm{H}$ ), 2.41 (s, 
3H), $2.93(\mathrm{t}, J 6.9 \mathrm{~Hz}, 2 \mathrm{H}), 7.23(\mathrm{~d}, J 8.1 \mathrm{~Hz}, 2 \mathrm{H}), 7.96$ (d, $J 8.1 \mathrm{~Hz}, 2 \mathrm{H}) .{ }^{13} \mathrm{C} \mathrm{NMR}\left(75 \mathrm{MHz}, \mathrm{CDCl}_{3}\right) ; \delta 14.1,22.7$, 26.7, 29.0, 29.3, 29.5, 29.6, 31.9, 125.4, 128.7, 129.1, 137.2, 167.4, 180.3. Anal. Calc. for $\mathrm{C}_{26} \mathrm{H}_{42} \mathrm{~N}_{2} \mathrm{O}$ C, 78.34; H, 10.62; N, 7.03; Found: C, 78.68; H, 10.58, N, 7.21\%.

3-(4-Chlorophenyl)-5-heptadecanyl-1,2,4-oxadiazole (6n)<smiles></smiles>

Yield: 79\%; colorless crystals; mp $53{ }^{\circ} \mathrm{C}$; $\mathrm{R}_{\mathrm{f}} 0.53$. $\left(\mathrm{CHCl}_{3}: \mathrm{C}_{6} \mathrm{H}_{12}, 1: 1\right)$. IR (KBr) $v_{\max } / \mathrm{cm}^{-1}: 3048,2925,2925$, 2847, 1574. ${ }^{1} \mathrm{H}$ NMR $\left(300 \mathrm{MHz}, \mathrm{CDCl}_{3}\right) \delta 8.01(\mathrm{~d}, J 8.7 \mathrm{~Hz}$, 2H), 7.45 (d, J 8.7 Hz, 2H), 2.94 (t, J 8.1 Hz, 2H), 1.86 (quintet, $J 8.1 \mathrm{~Hz}, 2 \mathrm{H}), 1.25(\mathrm{~m}, 28 \mathrm{H}), 0.88(\mathrm{t}, J 6.6 \mathrm{~Hz}$, $3 \mathrm{H}) .{ }^{13} \mathrm{C}$ NMR (75 MHz, $\left.\mathrm{CDCl}_{3}\right) ; \delta 14.1,22.7,26.6,29.0$, 29.1, 29.3, 29.5, 29.6, 29.64, 29.67, 31.9, 125.4, 128.7, 129.1, 137.2, 167.4, 180.3. Anal. Calc. for $\mathrm{C}_{25} \mathrm{H}_{39} \mathrm{ClN}_{2} \mathrm{O}$ C, 71.66; H, 9.38; N, 6.69. Found: C, 72.01; H, 9.47; N, $6.98 \%$.

5-Pentadecanyl-3-p-tolyl-1,2,4-oxadiazole (6o)

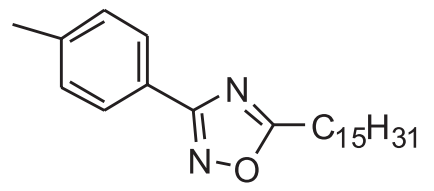

Yield: 90\%; colorless crystals; mp $57{ }^{\circ} \mathrm{C}$ (lit, ${ }^{2} \mathrm{mp}$ $\left.57.1{ }^{\circ} \mathrm{C}\right) ; \mathrm{R}_{\mathrm{f}} 0.51\left(\mathrm{CHCl}_{3}: \mathrm{C}_{6} \mathrm{H}_{12}, 1: 1\right)$. IR $(\mathrm{KBr}) \mathrm{v}_{\max } / \mathrm{cm}^{-1}$ : 3063, 2954, 2917, 2848, 1588. ${ }^{1} \mathrm{H} \mathrm{NMR}\left(300 \mathrm{MHz}, \mathrm{CDCl}_{3}\right)$; $\delta 0.88(\mathrm{t}, J 6.9 \mathrm{~Hz}, 3 \mathrm{H}), 1.25-1.44$ (bs, $24 \mathrm{H}$ ), 1.86 (quintet, $J 7.8 \mathrm{~Hz}, 2 \mathrm{H}), 2.41(\mathrm{~s}, 3 \mathrm{H}), 2.93(\mathrm{t}, J 7.8 \mathrm{~Hz}, 2 \mathrm{H}), 7.23$ (d, $J 8.1 \mathrm{~Hz}, 2 \mathrm{H}), 7.95(\mathrm{~d}, J 8.1 \mathrm{~Hz}, 2 \mathrm{H})$.

3,5-Di-p-tolyl-1,2,4-oxadiazole (6p)

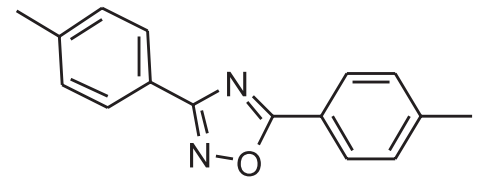

Yield: 75\%; colorless crystals; mp $133{ }^{\circ} \mathrm{C}$ (lit, ${ }^{2} \mathrm{mp} 134$ $\left.{ }^{\circ} \mathrm{C}\right) ; \mathrm{R}_{\mathrm{f}} 0.72\left(\mathrm{CHCl}_{3}\right)$. IR ( $\left.\mathrm{KBr}\right) \nu_{\max } / \mathrm{cm}^{-1}: 3023,2920,2850$, 1594. ${ }^{1} \mathrm{H}$ NMR (300 MHz, $\mathrm{CDCl}_{3}$ ); $\delta 2.39$ and 2.40 (2s, $6 \mathrm{H}), 7.27$ (d, J 7.6 Hz, 2H, ), 7.28 (d, J 7.8 Hz, 2H, ), 8.05 (d, J $7.8 \mathrm{~Hz}, 2 \mathrm{H}$, ), 8.06 (d, J 7.6 Hz, 2H, ).
3,5-Diphenyl-1,2,4-oxadiazole (6q)<smiles>c1ccc(-c2noc(-c3ccccc3)n2)cc1</smiles>

Yield: $86 \%$; colorless crystals; mp 108-109 ${ }^{\circ} \mathrm{C}$ (lit. ${ }^{2} \mathrm{mp}$ $\left.110{ }^{\circ} \mathrm{C}\right) ; \mathrm{R}_{\mathrm{f}} 0.70\left(\mathrm{CHCl}_{3}\right)$. IR ( $\left.\mathrm{KBr}\right) \nu_{\max } / \mathrm{cm}^{-1}: 3022,2920$, 2839, 1594. ${ }^{1} \mathrm{H}$ NMR $\left(300 \mathrm{MHz}, \mathrm{CDCl}_{3}\right) ; \delta 7.51-7.62(\mathrm{~m}$, $6 \mathrm{H}), 8.14-8.25(\mathrm{~m}, 4 \mathrm{H})$.

3-Phenyl-3-p-tolyl-1,2,4-oxadiazole (6r)<smiles>Cc1ccc(-c2noc(-c3ccccc3)n2)cc1</smiles>

Yield: 93\%; colorless crystals; $\mathrm{mp} 105-106{ }^{\circ} \mathrm{C}\left(\mathrm{lit},{ }^{2} \mathrm{mp}\right.$ $\left.105-106^{\circ} \mathrm{C}\right) ; \mathrm{R}_{\mathrm{f}} 0.52\left(\mathrm{CHCl}_{3} / \mathrm{C}_{6} \mathrm{H}_{12}, 1: 1\right)$. IR $(\mathrm{KBr}) v_{\max } / \mathrm{cm}^{-1}$ : 3049, 2955, 2915, 1560. ${ }^{1} \mathrm{H}$ NMR (300 MHz, $\left.\mathrm{CDCl}_{3}\right)$; $\delta 2.44$ (s, 3H), 7.32 (d, J 9.6 Hz, 2H), 7.55-7.60 (m, 3H), 8.07 (d, J 8.7 Hz, 2H), 8.22 (d, J 9.6 Hz, 2H).

5-Cyclohexyl-4-p-tolyl-1,2,4-oxadiazole (6s)<smiles>Cc1ccc(-c2noc(C3CCCCC3)n2)cc1</smiles>

Yield: $91 \%$; colorless crystals; mp $55{ }^{\circ} \mathrm{C} ; \mathrm{R}_{\mathrm{f}} 0.80$ $\left(\mathrm{CHCl}_{3}\right) . \mathrm{IR}(\mathrm{KBr}) \mathrm{v}_{\max } / \mathrm{cm}^{-1}: 3035,2918,2852,1589 .{ }^{1} \mathrm{H}$ NMR (300 MHz, $\left.\mathrm{CDCl}_{3}\right) ; \delta 1.25-2.15(\mathrm{~m}, 10 \mathrm{H}), 2.41(\mathrm{~s}$, $3 \mathrm{H}), 3.00\left(\mathrm{tt}, J_{a x-a x} 11.1 \mathrm{~Hz}, J_{a x-e q} 3.6 \mathrm{~Hz}, 1 \mathrm{H}\right), 7.27(\mathrm{~d}, J 8.4$ $\mathrm{Hz}, 2 \mathrm{H}), 7.96$ (d, J 8.4 Hz, 2H). ${ }^{13} \mathrm{C} \mathrm{NMR}\left(75 \mathrm{MHz} \mathrm{CDCl}_{3}\right)$; $\delta 21.5,25.3,25.5,30.2,36.3,124.2,127.3,129.4,141.2$, 168.0, 182.7. Anal. Calc. for $\mathrm{C}_{15} \mathrm{H}_{18} \mathrm{~N}_{2} \mathrm{O} \mathrm{C}, 74.35 ; \mathrm{H}, 7.49$; N, 11.56; Found: C, 74.48; H, 7.33; N, 11.62.

\section{1,2-bis(3-p-tolyl-1,2,4-oxadiazol-5-yl)ethane (6t)}<smiles>Cc1ccc(-c2noc(CCc3nc(-c4ccc(C)cc4)no3)n2)cc1</smiles>

Yield: $84 \%$; colorless crystals; mp $169-170{ }^{\circ} \mathrm{C}$ (lit. ${ }^{2}$ mp 171-172 $\left.{ }^{\circ} \mathrm{C}\right) ; \mathrm{R}_{\mathrm{f}} 0.73\left(\mathrm{CHCl}_{3}\right)$. IR $(\mathrm{KBr}) \mathrm{v}_{\max } / \mathrm{cm}^{-1}$ : 3032, 2926, 2854, 1590. ${ }^{1} \mathrm{H}$ NMR (300 MHz, $\left.\mathrm{CDCl}_{3}\right) ; \delta$ 
$2.41(\mathrm{~s}, 6 \mathrm{H}), 3.57$ (s, 4H), 7.28 (d, J $8.4 \mathrm{~Hz}, 4 \mathrm{H}), 7.95$ (d, $J 8.4 \mathrm{~Hz}, 4 \mathrm{H})$.

\section{References}

1. Srivastava, R. M.; Brinn, I. M.; Machuca-Herrera, J. O.; Faria, H. B.; Carpenter, D. G. B.; Venkatesh, C. G.; de Morais, L. P. F.; J. Mol. Struct. 1997, 406, 159.
2. For compounds 6o-r see: Bezerra, N. M. M.; Oliveira, S. P.; Srivastava, R. M.; da Silva, J. R.; Il Farmaco 2005, 60, 955. Leite, L. F. C.; Srivastava, R. M.; Cavalcanti, A. P.; Bull. Soc. Chim. Belges 1989, 98, 203. Goetz, N.; Synthesis 1976, 4, 268. Chiou, S.; J. Heterocycl. Chem. 1989, 26, 125. For compound 6t see: Srivastava, R. M.; da Silva, A. J. C. N.; de Oliveira, M. L.; J. Braz. Chem. Soc. 1993, 4, 84.

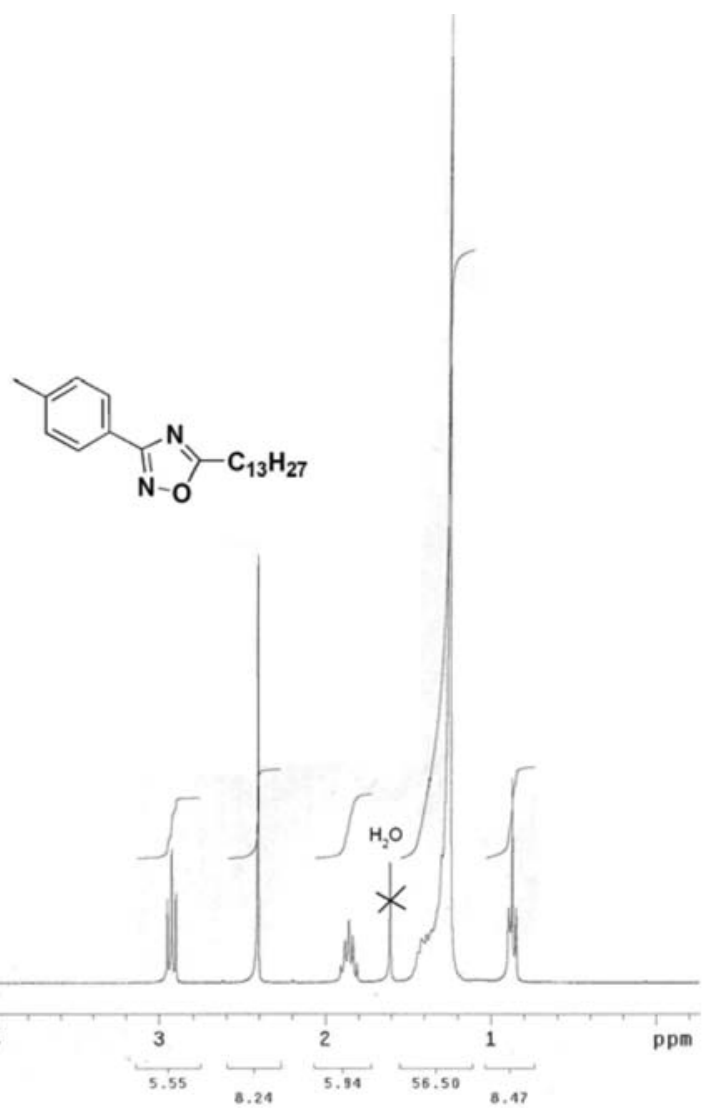

Figure S1. ${ }^{1} \mathrm{H}$ NMR (300 MHz) spectrum of compound $\mathbf{6} \mathbf{k}$ in $\mathrm{CDCl}_{3}$. 

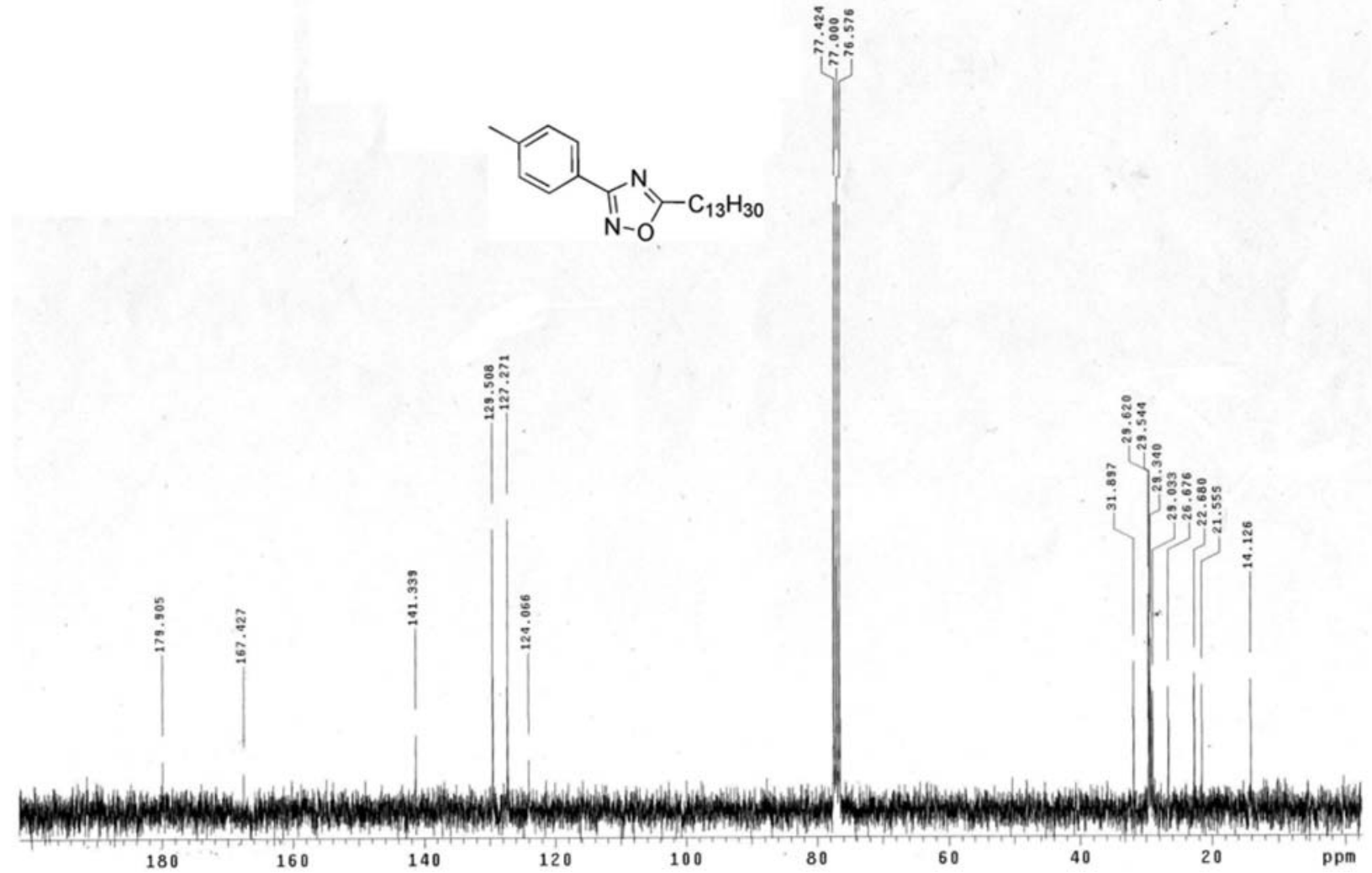

Figure S2. ${ }^{13} \mathrm{C}$ NMR (75 MHz) spectrum of compound $\mathbf{6 k}$ in $\mathrm{CDCl}_{3}$.

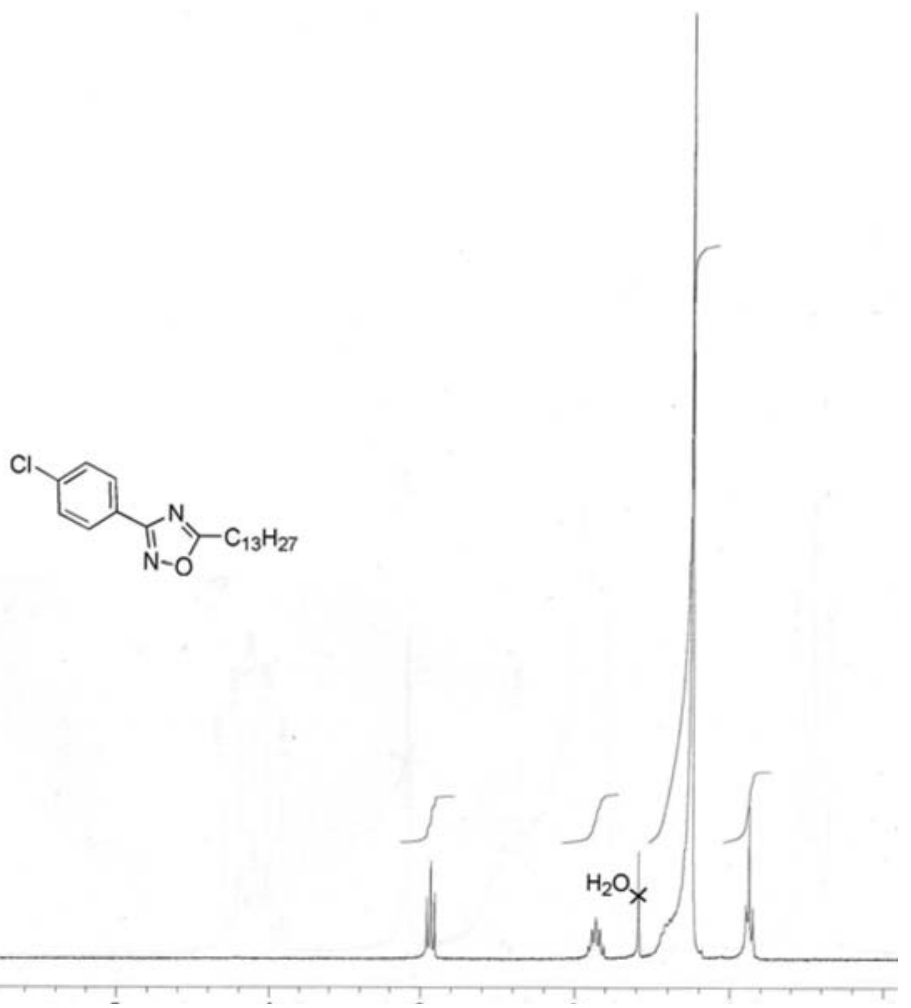

Figure S3. ${ }^{1} \mathrm{H}$ NMR (300 MHz) spectrum of compound 61 in $\mathrm{CDCl}_{3}$. 


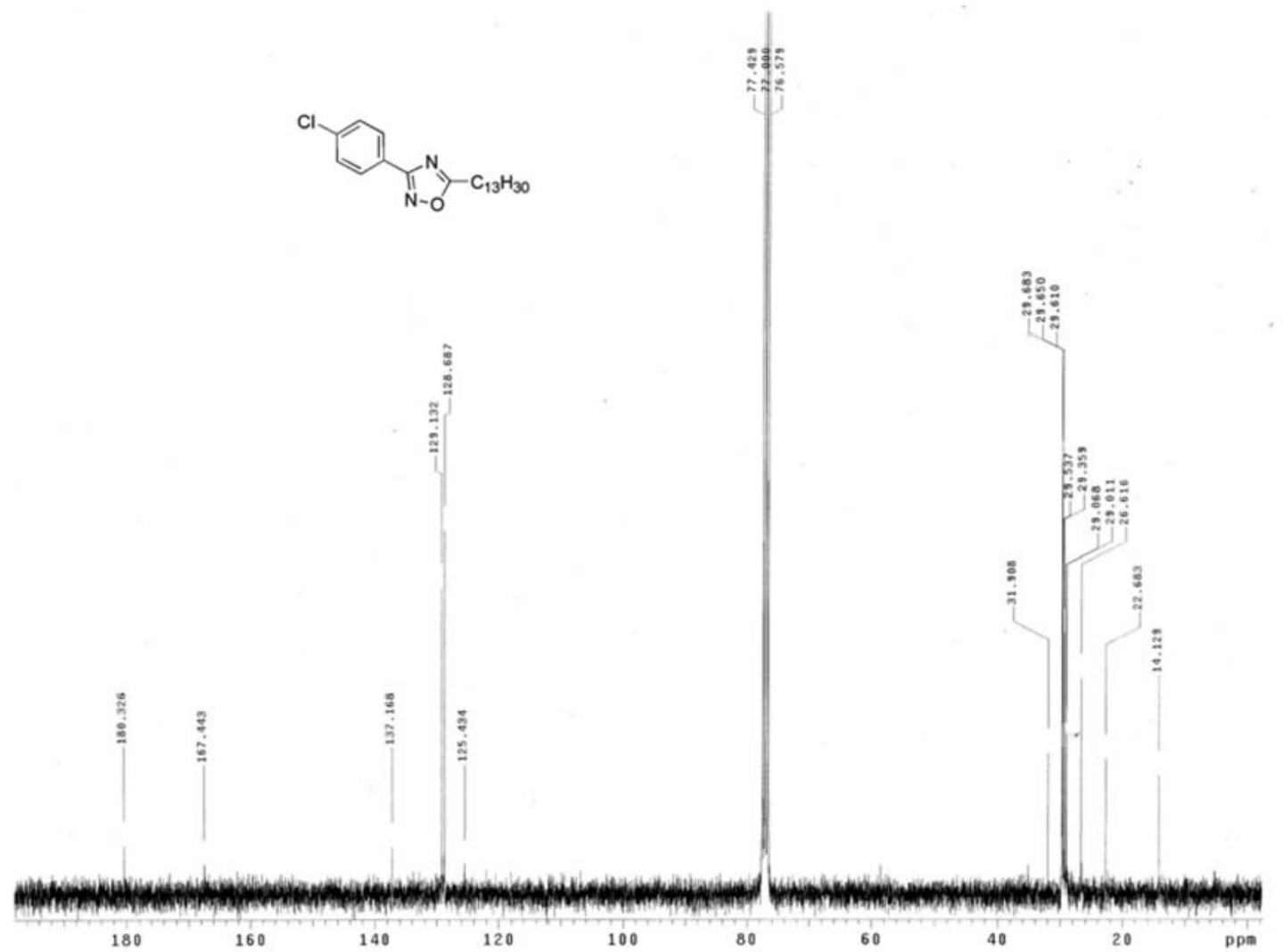

Figure S4. ${ }^{13} \mathrm{C}$ NMR (75 MHz) spectrum of compound 61 in $\mathrm{CDCl}_{3}$.

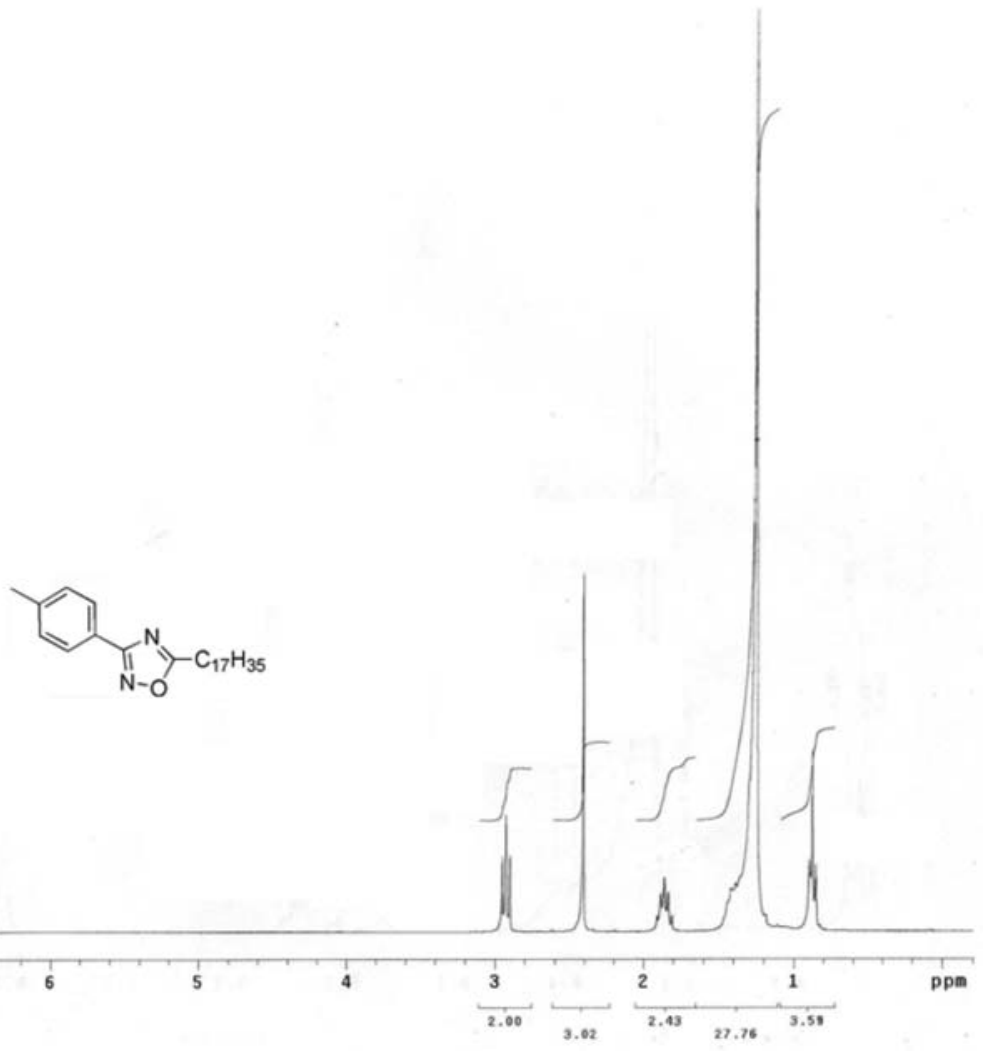

Figure S5. ${ }^{1} \mathrm{H}$ NMR (300 MHz) spectrum of compound $\mathbf{6 m}$ in $\mathrm{CDCl}_{3}$. 


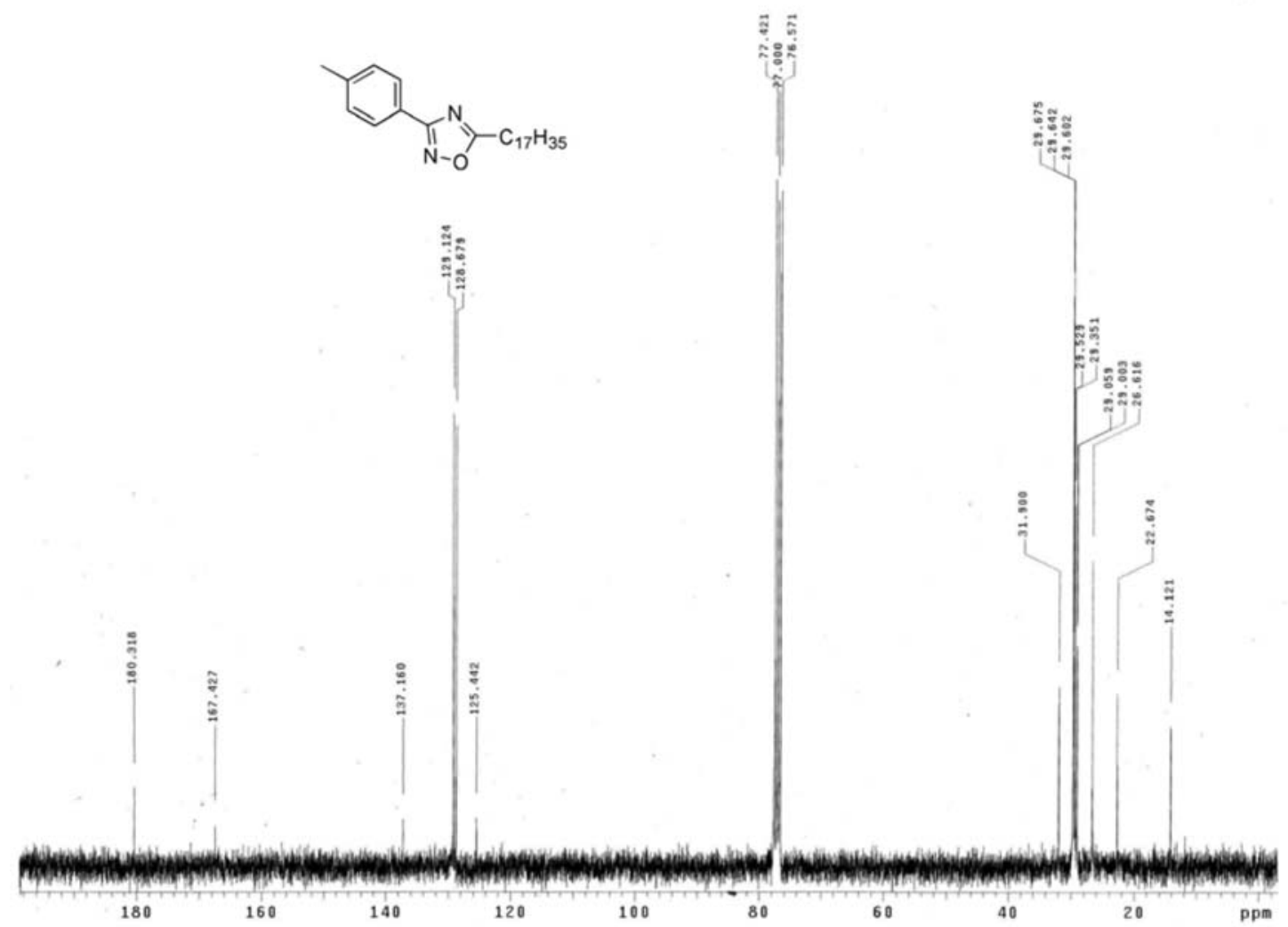

Figure S6. ${ }^{13} \mathrm{C} \mathrm{NMR}(75 \mathrm{MHz})$ spectrum of compound $\mathbf{6 m}$ in $\mathrm{CDCl}_{3}$.

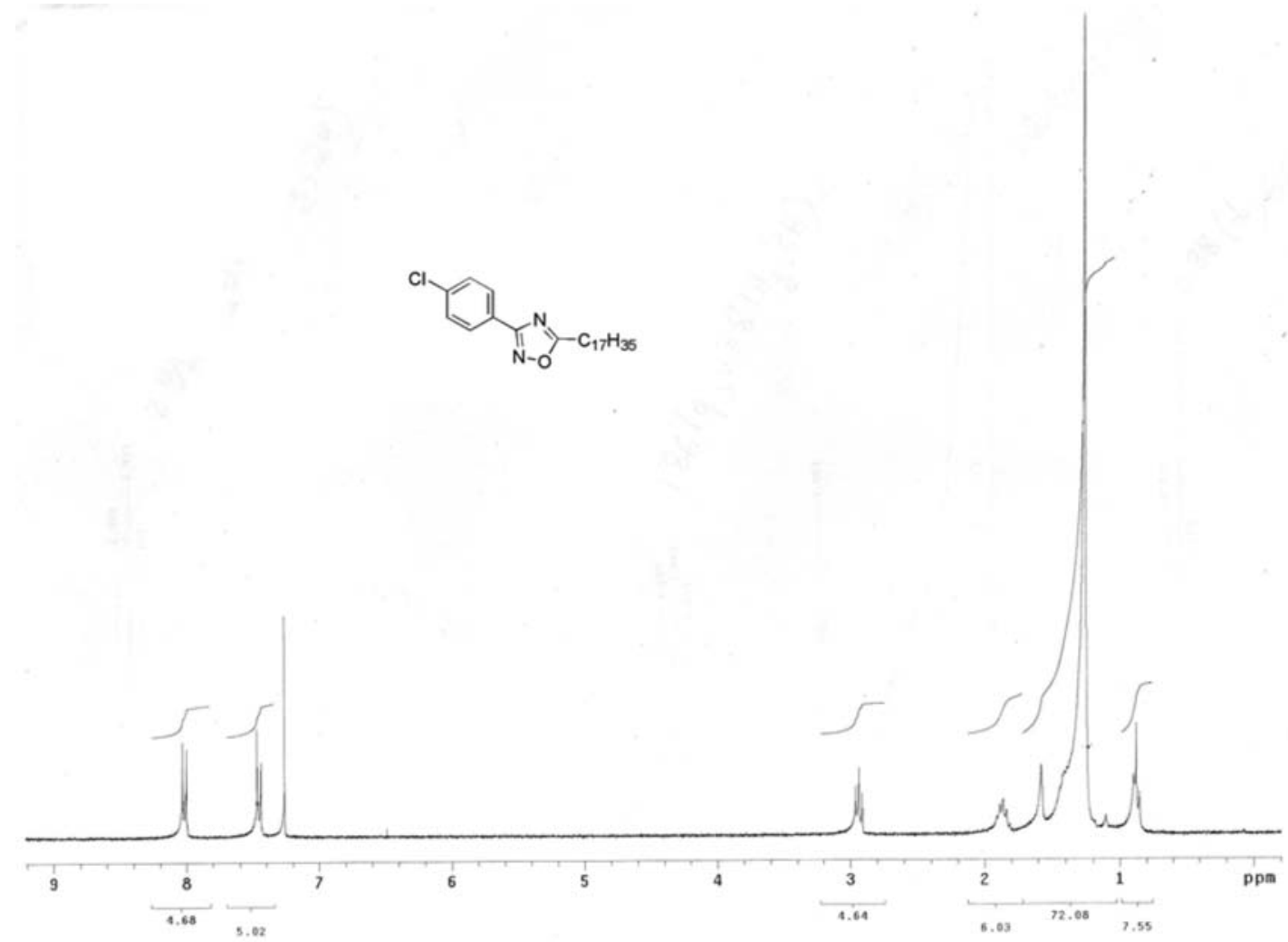

Figure S7. ${ }^{1} \mathrm{H}$ NMR (100 MHz) spectrum of compound $\mathbf{6 n}$ in $\mathrm{CDCl}_{3}$. 


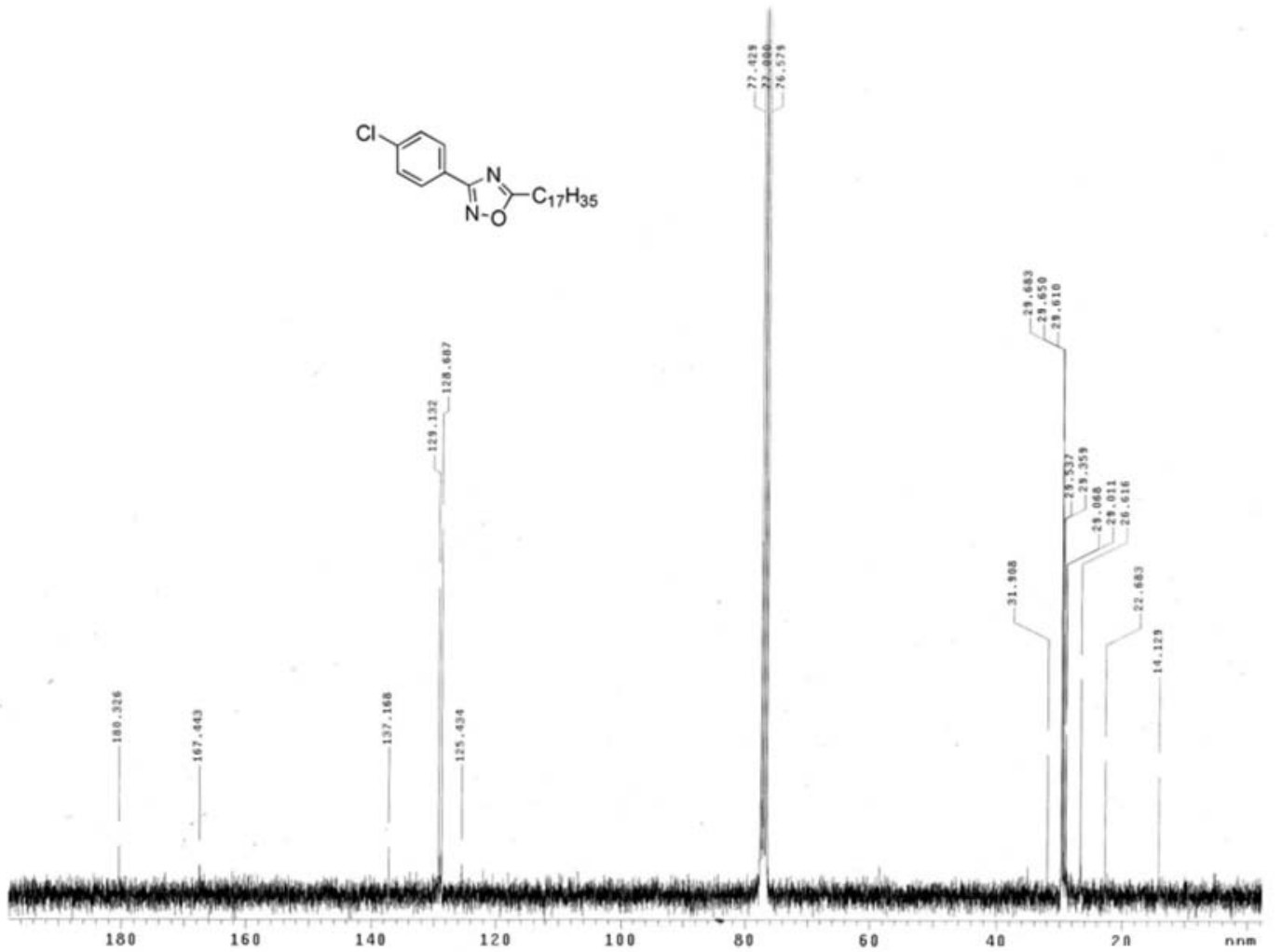

Figure S8. ${ }^{13} \mathrm{C}$ NMR (75 MHz) spectrum of compound $\mathbf{6 n}$ in $\mathrm{CDCl}_{3}$.

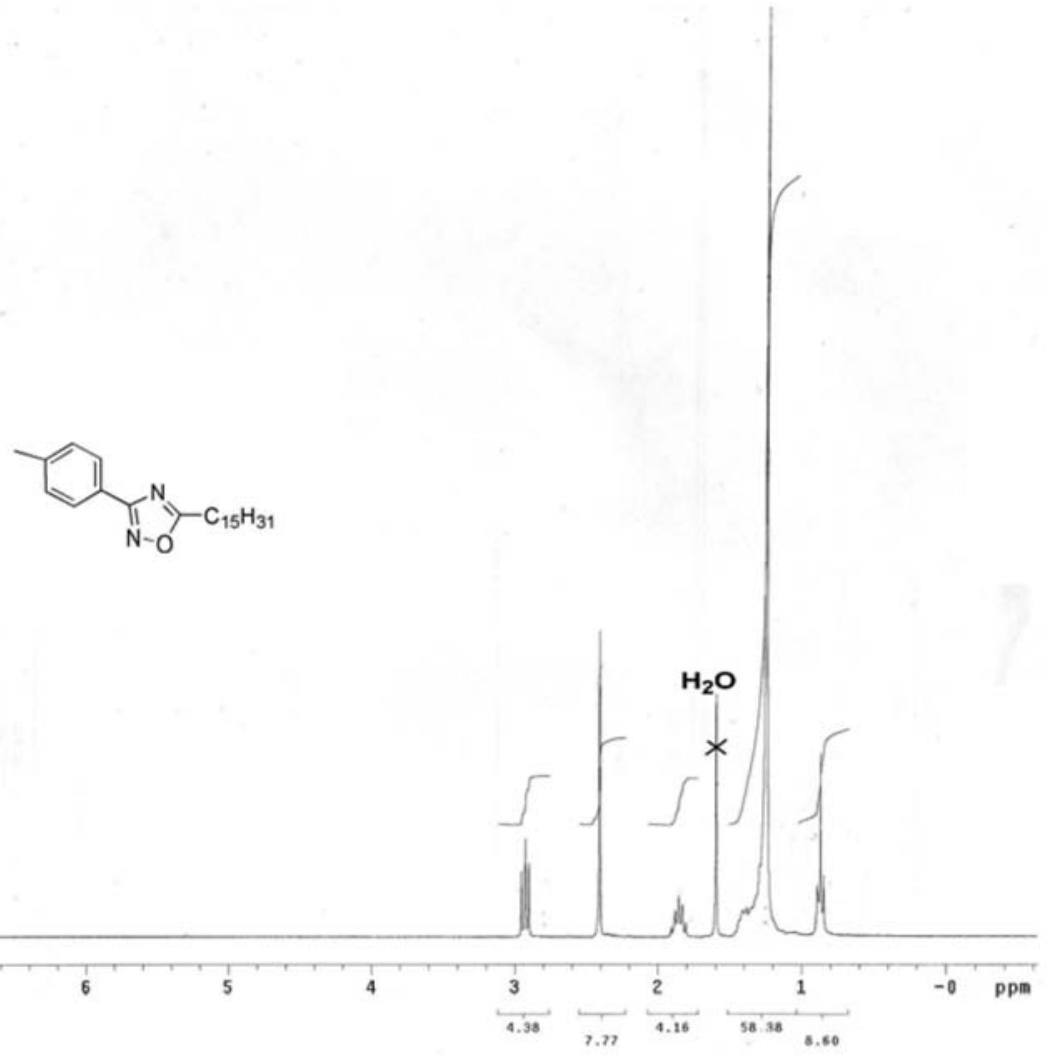

Figure S9. ${ }^{1} \mathrm{H}$ NMR (300 MHz) spectrum of compound 60 in $\mathrm{CDCl}_{3}$. 


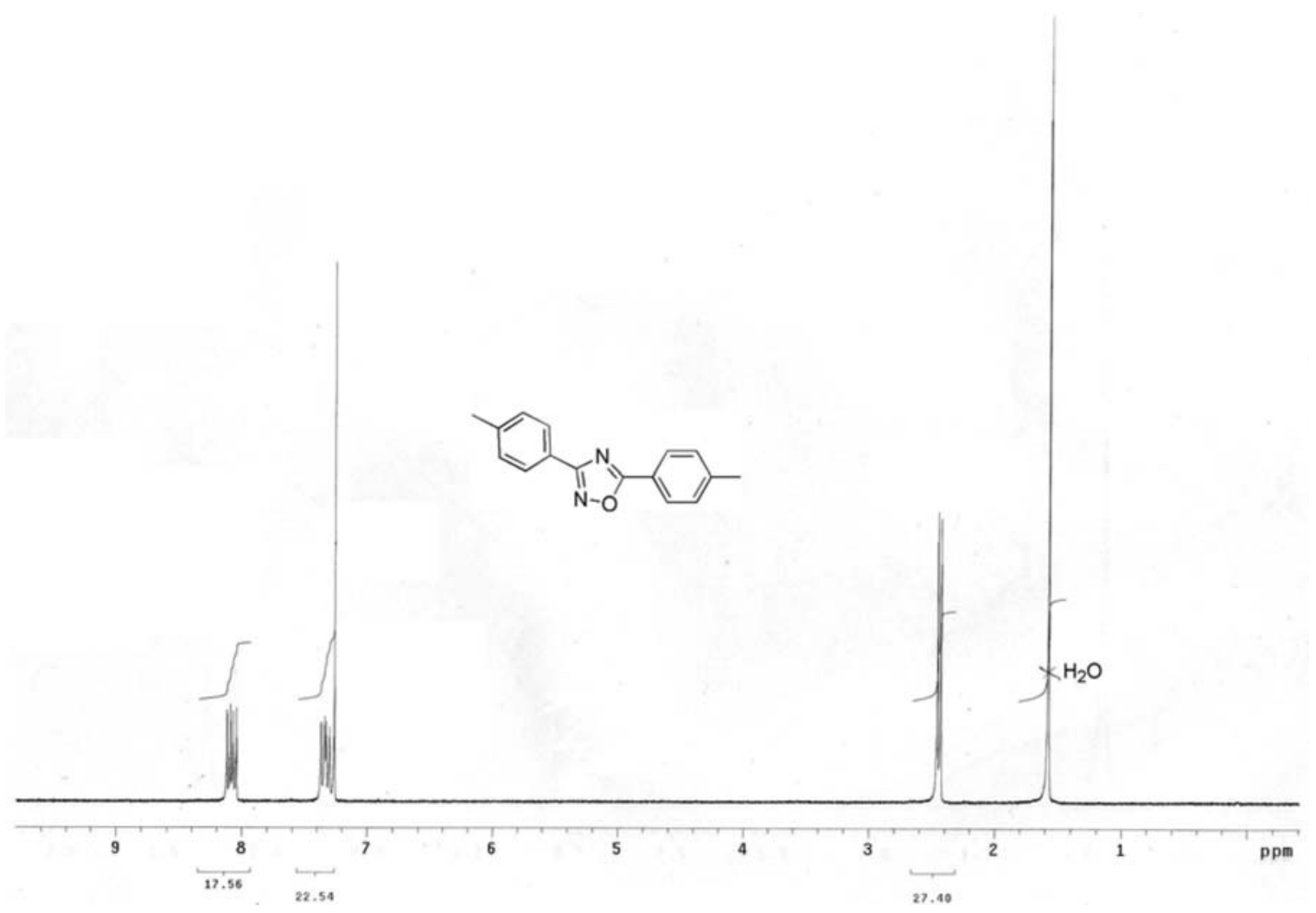

Figure S10. ${ }^{1} \mathrm{H}$ NMR $(300 \mathrm{MHz})$ spectrum of compound $\mathbf{6 p}$ in $\mathrm{CDCl}_{3}$.

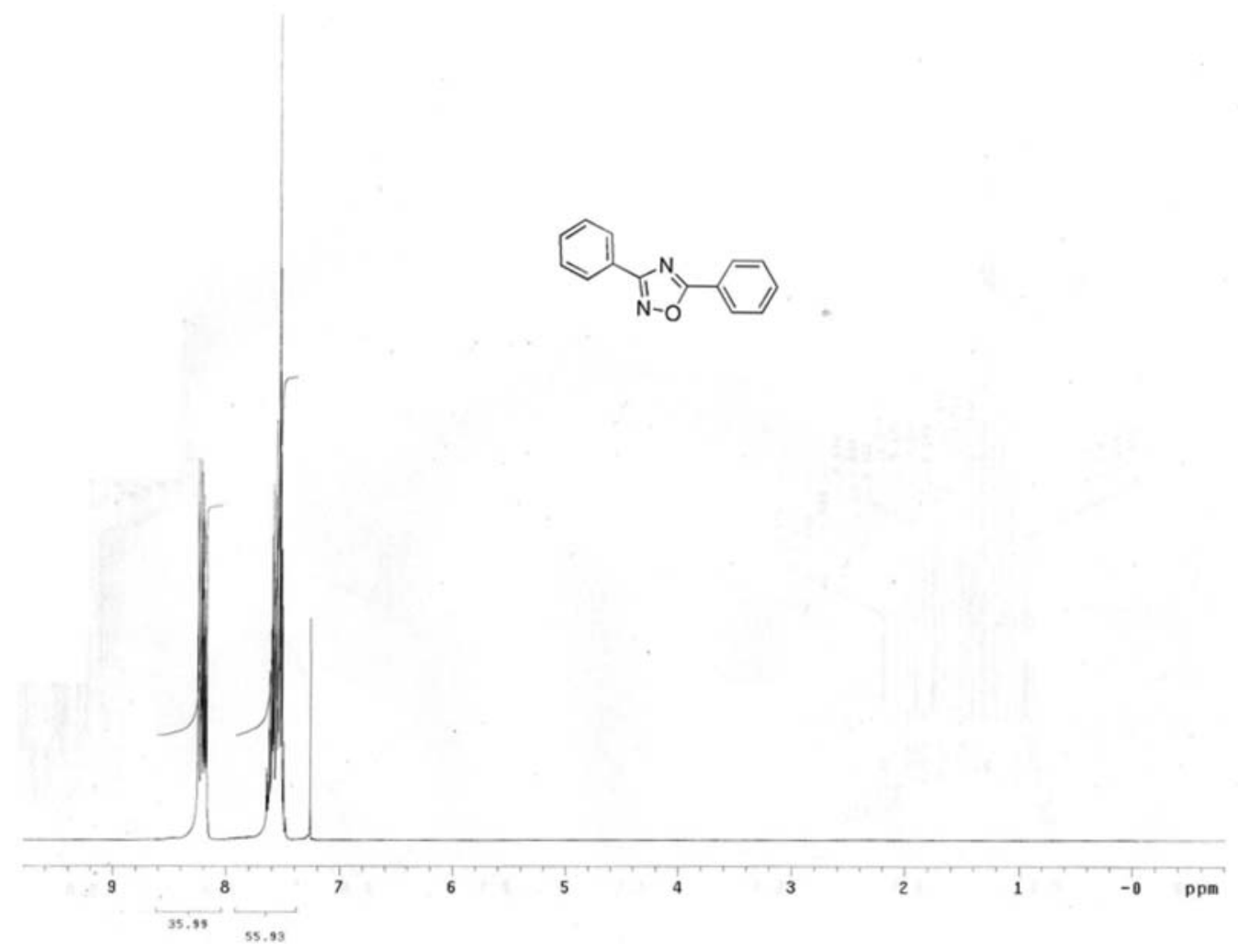

Figure S11. ${ }^{1} \mathrm{H}$ NMR $(300 \mathrm{MHz})$ spectrum of compound $\mathbf{6 q}$ in $\mathrm{CDCl}_{3}$. 


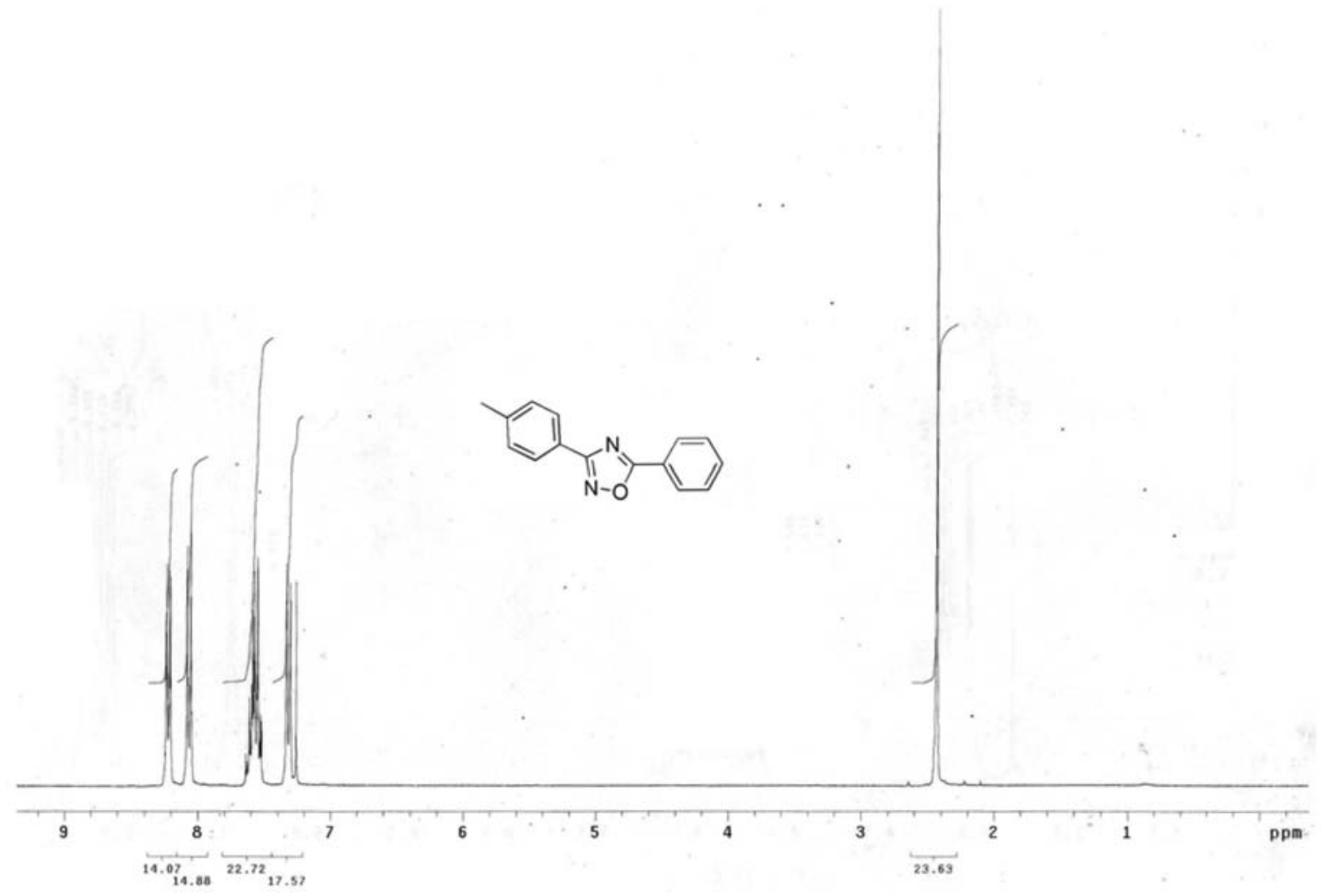

Figure S12. ${ }^{1} \mathrm{H}$ NMR (300 MHz) spectrum of compound $\mathbf{6 r}$ in $\mathrm{CDCl}_{3}$.

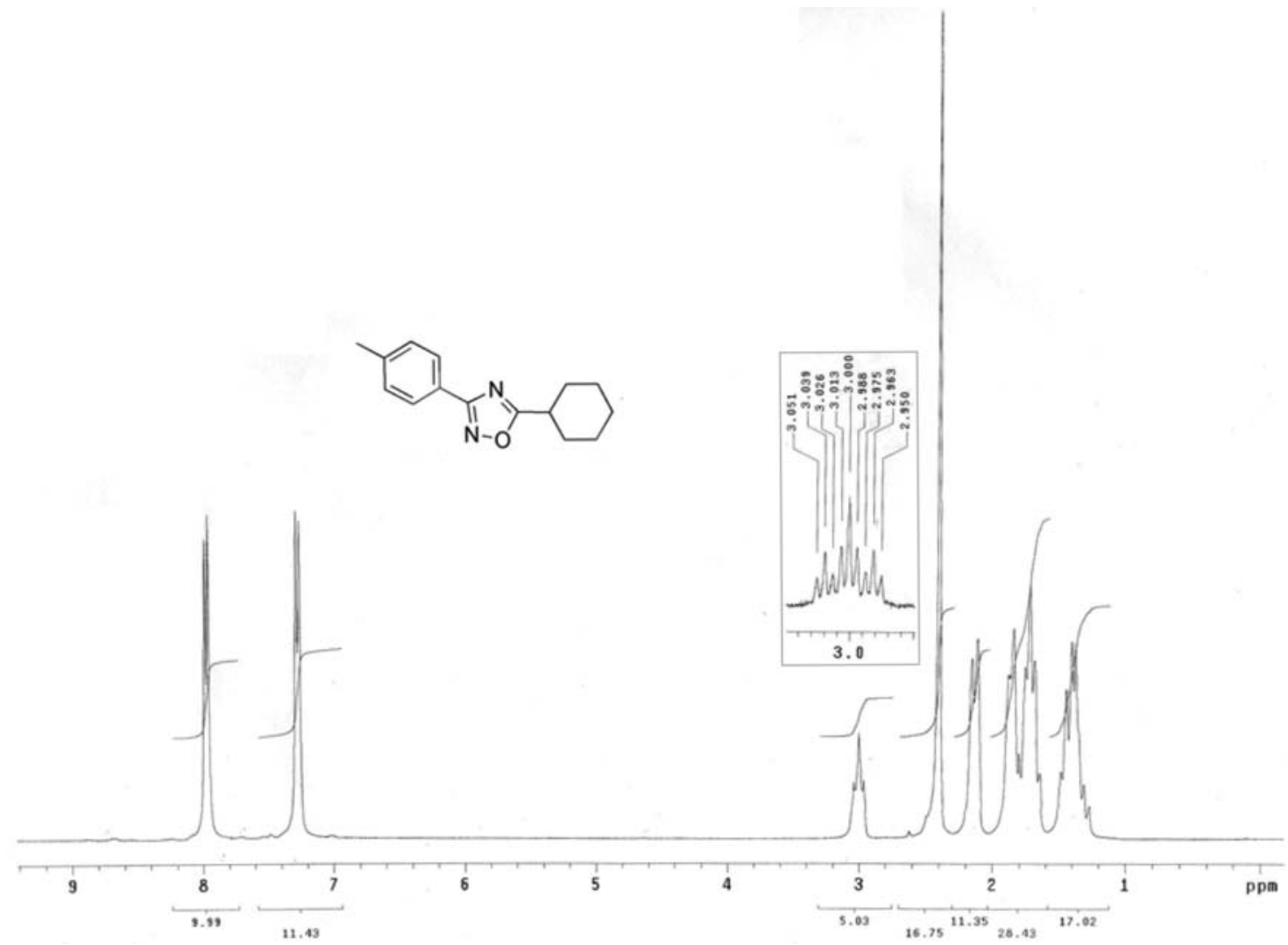

Figure S13. ${ }^{1} \mathrm{H}$ NMR (300 MHz) spectrum of compound $\mathbf{6 s}$ in $\mathrm{CDCl}_{3}$. 


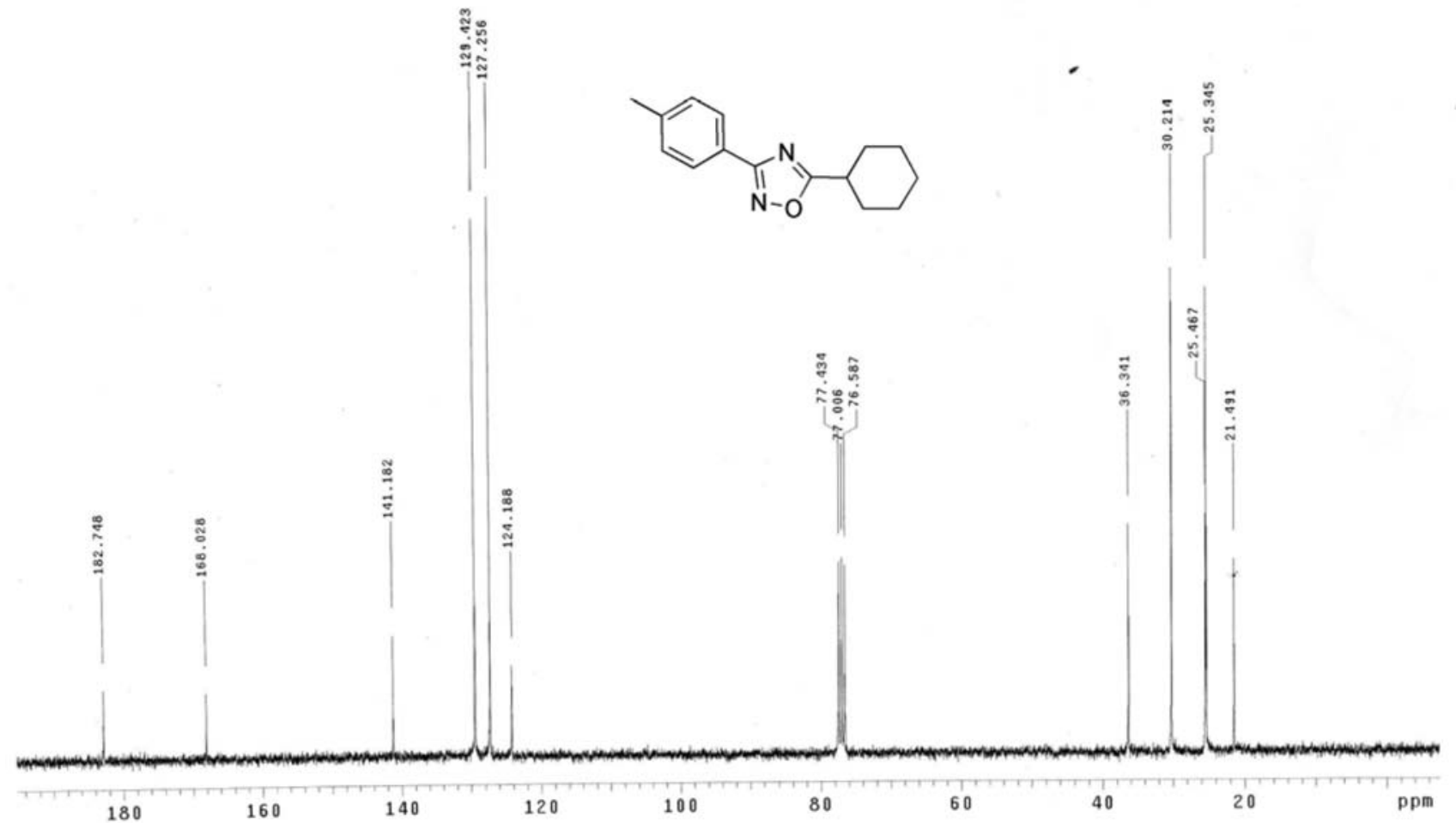

Figure S14. ${ }^{13} \mathrm{C}$ NMR $(75 \mathrm{MHz})$ spectrum of compound 6 s in $\mathrm{CDCl}_{3}$.

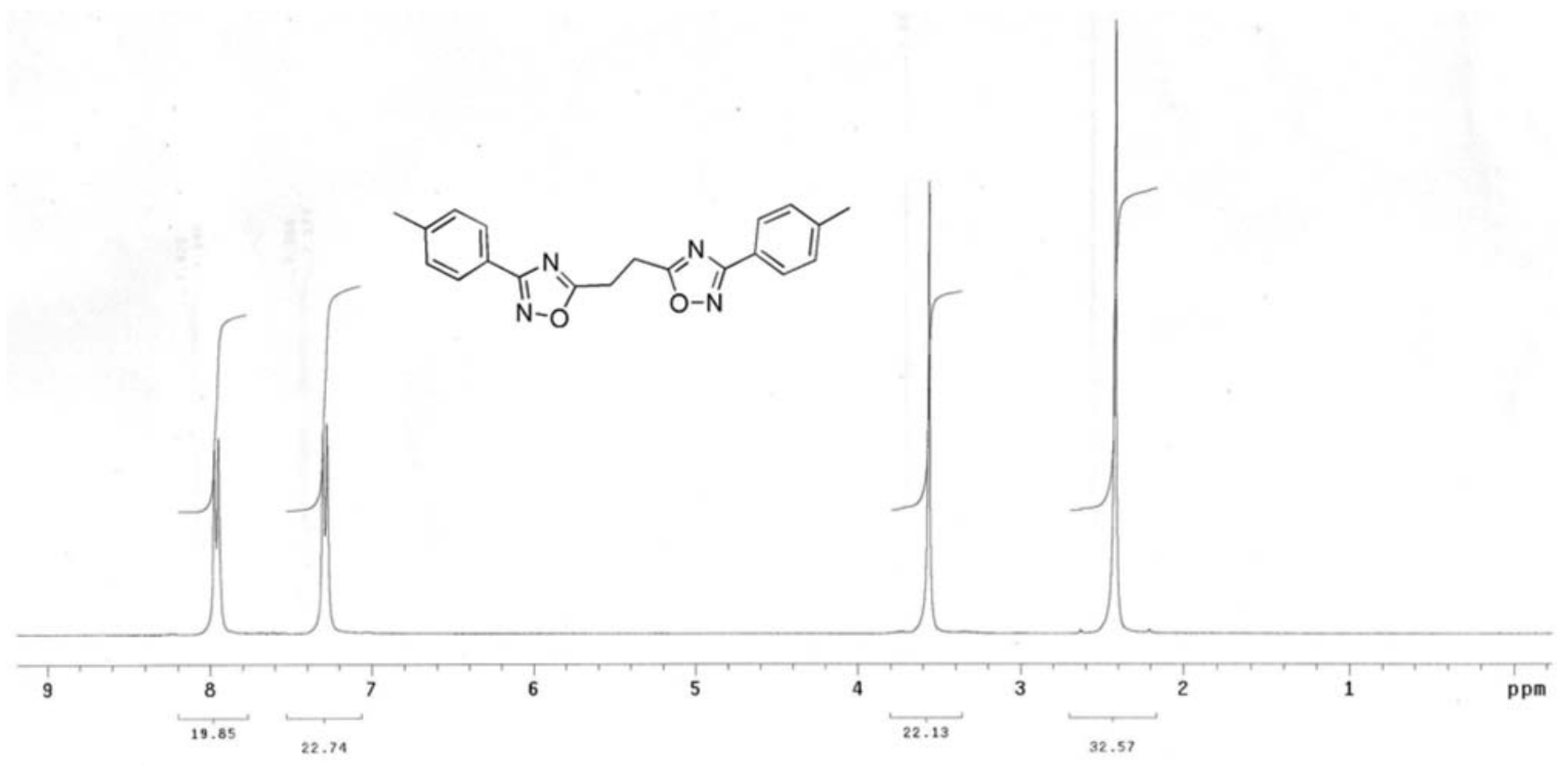

Figure S15. ${ }^{1} \mathrm{H}$ NMR (300 MHz) spectrum of compound $\mathbf{6 t}$ in $\mathrm{CDCl}_{3}$. 